\title{
A Survey on Oscillation of Impulsive Ordinary Differential Equations
}

\author{
Ravi P. Agarwal, ${ }^{1,2}$ Fatma Karakoç, ${ }^{3}$ and Ağacık Zafer ${ }^{4}$ \\ ${ }^{1}$ Department of Mathematical Sciences, FL Institute of Technology, Melbourne, FL 32901, USA \\ ${ }^{2}$ Mathematics and Statistics Department, King Fahd University of Petroleum and Minerals, \\ Dhahran 31261, Saudi Arabia \\ ${ }^{3}$ Department of Mathematics, Faculty of Sciences, Ankara University, 06100 Ankara, Turkey \\ ${ }^{4}$ Department of Mathematics, Middle East Technical University, 06531 Ankara, Turkey
}

Correspondence should be addressed to Ravi P. Agarwal, agarwal@fit.edu

Received 1 December 2009; Accepted 3 March 2010

Academic Editor: Leonid Berezansky

Copyright (c) 2010 Ravi P. Agarwal et al. This is an open access article distributed under the Creative Commons Attribution License, which permits unrestricted use, distribution, and reproduction in any medium, provided the original work is properly cited.

This paper summarizes a series of results on the oscillation of impulsive ordinary differential equations. We consider linear, half-linear, super-half-linear, and nonlinear equations. Several oscillation criteria are given. The Sturmian comparison theory for linear and half linear equations is also included.

\section{Introduction}

Impulsive differential equations, that is, differential equations involving impulse effect, appear as a natural description of observed evolution phenomena of several real world problems. There are many good monographs on the impulsive differential equations [16]. It is known that many biological phenomena, involving thresholds, bursting rhythm models in medicine and biology, optimal control models in economics, pharmacokinetics, and frequency modulates systems, do exhibit impulse effects. Let us describe the Kruger-Thiemer model [7] for drug distribution to show how impulses occur naturally. It is assumed that the drug, which is administered orally, is first dissolved into the gastrointestinal tract. The drug is then absorbed into the so-called apparent volume of distribution and finally eliminated from the system by the kidneys. Let $x(t)$ and $y(t)$ denote the amounts of drug at time $t$ in the gastrointestinal tract and apparent volume of distribution, respectively, and let $k_{1}$ and $k_{2}$ be the relevant rate constants. For simplicity, assume that $k_{1} \neq k_{2}$. The dynamic description of this model is then given by

$$
\begin{gathered}
x^{\prime}=-k_{1} x, \\
y^{\prime}=-k_{2} y+k_{1} x .
\end{gathered}
$$


In [8], the authors postulate the following control problem. At discrete instants of time $0=$ $t_{0}<t_{1}<t_{2}<\cdots<t_{N}$, the drug is ingested in amounts $\delta_{0}, \delta_{1}, \delta_{2}, \ldots, \delta_{N}$. This imposes the following boundary conditions:

$$
\begin{gathered}
x\left(t_{i}^{+}\right)=x\left(t_{i}^{-}\right)+\delta_{i}, \quad i=0,1,2, \ldots, N, \\
y\left(t_{i}^{+}\right)=y\left(t_{i}^{-}\right), \\
x\left(0^{-}\right)=y\left(0^{-}\right)=0 .
\end{gathered}
$$

To achieve the desired therapeutic effect, it is required that the amount of drug in the apparent volume of distribution never goes below a constant level or plateau $m$, say, during the time interval $\left[t_{1}, t_{N+1}\right]$, where $t_{N+1}>t_{N}$. Thus, we have the constraint

$$
y(t) \geq m, \quad t \in\left[t_{1}, t_{N+1}\right]
$$

It is also assumed that only nonnegative amounts of the drug can be given. Then, a control vector is a point $u=\left(\delta_{1}, \delta_{2}, \ldots, \delta_{N}\right)$ in the nonnegative orthant of Euclidean space of dimension $N+1$. Hence, $\Omega=\left\{u \in \mathbb{R}^{N+1}: u \geq \theta\right\}$. Finally, the biological cost function $f(\delta)=(1 / 2) \sum_{i=0}^{N} \delta_{i}^{2}$ minimizes both the side effects and the cost of the drug. The problem is to find $\inf _{u \geq \theta} f(u)$ subject to (1.1)-(1.3).

The first investigation on the oscillation theory of impulsive differential equations was published in 1989 [9]. In that paper Gopalsamy and Zhang consider impulsive delay differential equations of the form

$$
\begin{gathered}
x^{\prime}(t)+a x(t-\tau)=\sum_{j=1}^{\infty} b_{j} x\left(t_{j}^{-}\right) \delta\left(t-t_{j}\right), \quad t \neq t_{j}, \\
x^{\prime}(t)+p(t) x(t-\tau)=0, \quad t \neq t_{i}, \\
x\left(t_{i}^{+}\right)-x\left(t_{i}^{-}\right)=b_{i} x\left(t_{i}^{-}\right), \quad i \in \mathbb{N}=\{1,2, \ldots\},
\end{gathered}
$$

where $0<t_{1}<t_{2}<\cdots<t_{j} \rightarrow \infty$ as $j \rightarrow \infty$, and $\tau$ is a positive real number. Sufficient conditions are obtained for the asymptotic stability of the zero solution of (1.4) and existence of oscillatory solutions of (1.5). However, it seems that the problem of oscillation of ordinary differential equations with impulses has received attention much later [10]. Although, the theory of impulsive differential equations has been well established, the oscillation theory of such equations has developed rather slowly. To the best of our knowledge, except one paper [11], all of the investigations have been on differential equations subject to fixed moments of impulse effect. In [11], second-order differential equations with random impulses were dealt with, and there are no papers on the oscillation of differential equations with impulses at variable times.

In this survey paper, our aim is to present the results (within our reach) obtained so far on the oscillation theory of impulsive ordinary differential equations. The paper is organized as follows. Section 2 includes notations, definitions, and some well-known oscillation theorems needed in later sections. In Section 3, we are concerned with linear impulsive differential equations. In Section 4, we deal with nonlinear impulsive differential equations. 


\section{Preliminaries}

In this section, we introduce notations, definitions, and some well-known results which will be used in this survey paper.

Let $J=\left[t_{0}, \infty\right)$ for some fixed $t_{0} \geq 0$ and $\left\{t_{k}\right\}_{k=1}^{\infty}$ be a sequence in $J$ such that $t_{0} \leq t_{1}<$ $t_{2}<\cdots<t_{k}<t_{k+1}<\cdots$ and $\lim _{k \rightarrow \infty} t_{k}=\infty$.

By PLC $(X, Y)$, we denote the set of all functions $\psi: X \rightarrow Y$ which are continuous for $t \neq t_{k}$ and continuous from the left with discontinuities of the first kind at $t=t_{k}$. Similarly, $\operatorname{PLC}^{r}(X, Y)$ is the set of functions $\psi: X \rightarrow Y$ having derivative $\psi^{(r)} \in \operatorname{PLC}(X, Y)$. One has $X \subset J, Y=\mathbb{R}^{n}$, or $Y=\mathbb{R}$. In case $Y=\mathbb{R}$, we simply write $\operatorname{PLC}(X)$ for $\operatorname{PLC}(X, \mathbb{R})$. As usual, $C(X, Y)$ denotes the set of continuous functions from $X$ to $Y$.

Consider the system of first-order impulsive ordinary differential equations having impulses at fixed moments of the form

$$
\begin{gathered}
x^{\prime}=f(t, x), \quad t \neq t_{k}, \\
\left.\Delta x\right|_{t=t_{k}}=f_{k}(x), \quad k \in \mathbb{N},
\end{gathered}
$$

where $f(t, x) \in C\left(J \times \mathbb{R}^{n}, \mathbb{R}^{n}\right), f_{k} \in C\left(\mathbb{R}^{n}, \mathbb{R}^{n}\right)$, and

$$
\left.\Delta x\right|_{t=t_{k}}=x\left(t_{k}^{+}\right)-x\left(t_{k}^{-}\right)
$$

with $x\left(t_{k}^{ \pm}\right)=\lim _{t \rightarrow t_{k}^{ \pm}} x(t)$. The notation $\Delta x\left(t_{k}\right)$ in place of $\left.\Delta x\right|_{t=t_{k}}$ is also used. For simplicity, it is usually assumed that $x\left(t_{k}\right)=x\left(t_{k}^{-}\right)$.

The qualitative theory of impulsive ordinary differential equations of the form (2.1) can be found in $[1-6,12]$.

Definition 2.1. A function $\varphi \in \operatorname{PLC}^{1}\left(J, \mathbb{R}^{n}\right)$ is said to be a solution of $(2.1)$ in an interval $(\alpha, \beta)$ if $\varphi(t)$ satisfies (2.1) for $t \in(\alpha, \beta)$.

For $t_{0} \in(\alpha, \beta)$, we may impose the initial condition

$$
x\left(t_{0}^{+}\right)=x_{0} .
$$

Each solution $\varphi(t)$ of (2.1) which is defined in the interval $(\alpha, \beta)$ and satisfying the condition $\varphi\left(t_{0}^{+}\right)=x_{0}$ is said to be a solution of the initial value problem (2.1)-(2.3).

Note that if $t_{0} \in\left(t_{k}, t_{k+1}\right)$, then the solution of the initial value problem (2.1)-(2.3) coincides with the solution of

$$
x^{\prime}=f(t, x), \quad x\left(t_{0}\right)=x_{0}
$$

on $\left(t_{k}, t_{k+1}\right)$.

Definition 2.2. A real-valued function $x(t)$, not necessarily a solution, is said to be oscillatory, if it is neither eventually positive nor eventually negative. Otherwise, it is called nonoscillatory. A differential equation is called oscillatory if all its solutions are oscillatory. 
For our purpose we now state some well-known results on oscillation of second-order ordinary differential equations without impulses.

Theorem 2.3 (see [13]). Let $p(t) \geq 0$. Then, the equation

$$
y^{\prime \prime}+p(t) y=0
$$

is oscillatory if

$$
\liminf _{t \rightarrow \infty} \int_{t}^{\infty} p(s) d s>\frac{1}{4}
$$

and nonoscillatory if

$$
t \int_{t}^{\infty} p(s) d s \leq \frac{1}{4} \text { for large } t
$$

Theorem 2.4 (see [14]). Let $r$ and $p$ be continuous functions and $r(t), p(t)>0$. If

$$
\lim _{t \rightarrow \infty} \int_{1}^{t} \frac{d s}{r(s)}=\infty, \quad \lim _{t \rightarrow \infty} \int_{1}^{t} p(s) d s=\infty
$$

then the equation

$$
\left(r(t) y^{\prime}\right)^{\prime}+p(t) y=0
$$

is oscillatory.

Theorem 2.5 (see [15]). Let $f$ be a positive and continuously differentiable function for $t \geq 0$, and let $f^{\prime}(t) \leq 0$. If

$$
\int_{0}^{\infty} t^{2 n-1} f(t) d t<\infty
$$

then the equation

$$
y^{\prime \prime}+f(t) y^{2 n-1}=0
$$

has nonoscillatory solutions, where $n>1$ is an integer.

Theorem 2.6 (see [15]). Let $f(t)$ be a positive and continuous function for $t \geq 0$, and $n>1$ an integer. Then every solution of (2.11) is oscillatory if and only if

$$
\int_{0}^{\infty} t f(t) d t=\infty
$$




\section{Linear Equations}

In this section, we consider the oscillation problem for first-, second-, and higher-order linear impulsive differential equations. Moreover, the Sturm type comparison theorems for secondorder linear impulsive differential equations are included.

\subsection{Oscillation of First-Order Linear Equations}

Let us consider the linear impulsive differential equation

$$
\begin{gathered}
x^{\prime}+a(t) x=0, \quad t \neq t_{k}, \\
\left.\Delta x\right|_{t=t_{k}}+a_{k} x=0, \quad k \in \mathbb{N},
\end{gathered}
$$

together with the corresponding inequalities:

$$
\begin{gathered}
x^{\prime}+a(t) x \leq 0, \quad t \neq t_{k}, \\
\left.\Delta x\right|_{t=t_{k}}+a_{k} x \leq 0, \quad k \in \mathbb{N}, \\
x^{\prime}+a(t) x \geq 0, \quad t \neq t_{k}, \\
\left.\Delta x\right|_{t=t_{k}}+a_{k} x \geq 0, \quad k \in \mathbb{N} .
\end{gathered}
$$

The following theorems are proved in [1].

Theorem 3.1. Let $a \in \operatorname{PLC}(J)$ and $1-a_{k} \neq 0, k \in \mathbb{N}$. Then the following assertions are equivalent.

(1) The sequence $\left\{1-a_{k}\right\}$ has infinitely many negative terms.

(2) The inequality (3.2) has no eventually positive solution.

(3) The inequality (3.3) has no eventually negative solution.

(4) Each nonzero solution of (3.1) is oscillatory.

Proof. (1) $\Rightarrow(2)$. Let the sequence $\left\{1-a_{k}\right\}$ have infinitely many negative terms. Let us suppose that the assertion (2) is not true; that is, the inequality (3.2) has an eventually positive solution $x(t), t \geq T_{0}$. Let $t_{k} \geq T_{0}$ be such that $1-a_{k}<0$. Then, it follows from (3.2) that

$$
x\left(t_{k}^{+}\right) \leq\left(1-a_{k}\right) x\left(t_{k}\right)<0,
$$

which is a contradiction.

$(2) \Leftrightarrow(3)$. The validity of this relation follows from the fact that if $x(t)$ is a solution of the inequality (3.2), then $-x(t)$ is a solution of the inequality (3.3) and vice versa.

(2) and $(3) \Rightarrow(4)$. In fact, if (3.1) has neither an eventually positive nor an eventually negative solution, then each nonzero solution of (3.1) is oscillatory.

$(4) \Rightarrow(1)$. If $x(t)$ is an oscillatory solution of (3.3), then it follows from the equality

$$
x(t)=x\left(t_{0}\right) \exp \left(-\int_{t_{0}}^{t} a(s) d s\right) \prod_{t_{0} \leq t_{k}<t}\left(1-a_{k}\right)
$$

that the sequence $\left\{1-a_{k}\right\}$ has infinitely many negative terms. 
The following theorem can be proved similarly.

Theorem 3.2. Let $a \in \operatorname{PLC}(J)$, and $1-a_{k} \neq 0, k \in \mathbb{N}$. Then the following assertions are equivalent.

(1) The sequence $\left\{1-a_{k}\right\}$ has finitely many negative terms.

(2) The inequality (3.2) has an eventually positive solution.

(3) The inequality (3.3) has an eventually negative solution.

(4) Each nonzero solution of (3.1) is nonoscillatory.

It is known that (3.1) without impulses has no oscillatory solutions. But (3.1) (with impulses) can have oscillatory solutions. So, impulse actions determine the oscillatory properties of first-order linear differential equations.

\subsection{Sturmian Theory for Second-Order Linear Equations}

It is well-known that Sturm comparison theory plays an important role in the study of qualitative properties of the solutions of both linear and nonlinear equations. The first paper on the Sturm theory of impulsive differential equations was published in 1996. In [10], Bainov et al. derived a Sturmian type comparison theorem, a zeros-separation theorem, and a dichotomy theorem for second-order linear impulsive differential equations. Recently, the theory has been extended in various directions in [16-18], with emphasis on Picone's formulas, Wirtinger type inequalities, and Leighton type comparison theorems.

We begin with a series of results contained in [1, 10]. The second-order linear impulsive differential equations considered are

$$
\begin{gathered}
x^{\prime \prime}+p(t) x=0, \quad t \neq t_{k}, \\
\Delta x\left(t_{k}\right)=0, \quad \Delta x^{\prime}\left(t_{k}\right)+p_{k} x\left(t_{k}\right)=0, \quad k \in \mathbb{N}, \\
y^{\prime \prime}+q(t) y=0, \quad t \neq t_{k}, \\
\Delta y\left(t_{k}\right)=0, \quad \Delta y^{\prime}\left(t_{k}\right)+q_{k} y\left(t_{k}\right)=0, \quad k \in \mathbb{N},
\end{gathered}
$$

where $p$ and $q$ are continuous for $t \in J, t \neq t_{k}$, and they have a discontinuity of the first kind at the points $t_{k} \in J$, where they are continuous from the left.

The main result is the following theorem, which is also valid for differential inequalities.

Theorem 3.3 (see $[1,10]$ ). Suppose the following.

(1) Equation (3.7) has a solution $y(t)$ such that

$$
y(t)>0, \quad t \in(a, b), \quad y\left(a^{+}\right)=y\left(b^{-}\right)=0 .
$$

(2) The following inequalities are valid:

$$
p(t) \geq q(t), \quad p_{k} \geq q_{k}, \quad t \in(a, b), \quad t_{k} \in(a, b) .
$$

(3) $p(t)>q(t)$ in a subinterval of $(a, b)$ or $p_{k}>q_{k}$ for some $t_{k} \in(a, b)$.

Then (3.6) has no positive solution $x(t)$ defined on $(a, b)$. 
Proof. Assume that (3.6) has a solution $x(t)$ such that $x(t)>0, t \in(a, b)$. Then from the relation

$$
\left(x^{\prime}(t) y(t)-x(t) y^{\prime}(t)\right)^{\prime}=x^{\prime \prime}(t) y(t)-x(t) y^{\prime \prime}(t), \quad t \in(a, b), \quad t \neq t_{k},
$$

an integration yields

$$
\begin{aligned}
x^{\prime}\left(b^{-}\right) y\left(b^{-}\right)-x\left(b^{-}\right) y^{\prime}\left(b^{-}\right)-x^{\prime}\left(a^{+}\right) y\left(a^{+}\right)+x\left(a^{+}\right) y^{\prime}\left(a^{+}\right) \\
=\int_{a}^{b}\left[x^{\prime \prime}(t) y(t)-x(t) y^{\prime \prime}(t)\right] d t+\sum_{a<t_{k}<b}\left[\Delta x^{\prime}\left(t_{k}\right) y\left(t_{k}\right)-x\left(t_{k}\right) \Delta y^{\prime}\left(t_{k}\right)\right] .
\end{aligned}
$$

From (3.6), (3.7), condition (1), and the above inequality, we conclude that

$$
0 \leq \int_{a}^{b}(q(t)-p(t)) x(t) y(t) d t+\sum_{a<t_{k}<b}\left(q_{k}-p_{k}\right) x\left(t_{k}\right) y\left(t_{k}\right) .
$$

But, from conditions (2) and (3), it follows that the right side of the above inequality is negative, which leads to a contradiction. This completes the proof.

The following corollaries follow easily from Theorem 3.3.

Corollary 3.4 (Comparison Theorem). Suppose the following.

(1) Equation (3.7) has a solution $y(t)$ such that

$$
y(t) \neq 0, \quad t \in(a, b), \quad y\left(a^{+}\right)=y\left(b^{-}\right)=0 .
$$

(2) The following inequalities are valid:

$$
p(t) \geq q(t), \quad p_{k} \geq q_{k}, \quad t \in(a, b), \quad t_{k} \in(a, b) .
$$

(3) $p(t)>q(t)$ in some subinterval of $(a, b)$ or $p_{k}>q_{k}$ for some $t_{k} \in(a, b)$.

Then, each solution $x(t)$ of (3.6) has at least one zero in $(a, b)$.

Corollary 3.5. If conditions (1) and (2) of Corollary 3.4 are satisfied, then one has the following.

(1) Each solution $x(t)$ of (3.6) for which $\left|x\left(a^{+}\right)\right|+\left|x\left(b^{-}\right)\right|>0$ has at least one zero in $(a, b)$.

(2) Each solution $x(t)$ of (3.6) has at least one zero in $[a, b]$.

Corollary 3.6 (Oscillation Theorem). Suppose the following.

(1) There exists a solution $y(t)$ of (3.7) and a sequence of disjoint intervals $\left(a_{n}, b_{n}\right) \subset J$ such that

$$
\lim _{n \rightarrow \infty} a_{n}=\infty, \quad y\left(a_{n}^{+}\right)=y\left(b_{n}^{-}\right)=0, \quad \text { and } y(t) \neq 0
$$

for $t \in\left(a_{n}, b_{n}\right), n \in \mathbb{N}$. 
(2) The following inequalities are valid for $t \in\left(a_{n}, b_{n}\right), t_{k} \in\left(a_{n}, b_{n}\right)$, and $n \in \mathbb{N}$;

$$
p(t) \geq q(t), \quad p_{k} \geq q_{k} .
$$

Then all solutions of (3.6) are oscillatory, and moreover, they change sign in each interval $\left[a_{n}, b_{n}\right]$.

Corollary 3.7 (Comparison Theorem). Let the inequalities $p(t) \geq q(t), p_{k} \geq q_{k}$ hold for $t>T \geq \alpha$ and $t_{k}>T$. Then, all solutions of (3.7) are nonoscillatory if (3.6) has a nonoscillatory solution.

Corollary 3.8 (Separation Theorem). The zeros of two linearly independent solutions of (3.6) separate one another; that is, the two solutions have no common zeros, and if $a, b(a<b)$ are two consecutive zeros of one of the solutions, then the interval $(a, b)$ contains exactly one zero of the other solution.

Corollary 3.9 (Dichotomy Theorem). All solutions of (3.6) are oscillatory or nonoscillatory.

We can use Corollary 3.7, to deduce the following oscillation result for the equation:

$$
\begin{gathered}
z^{\prime \prime}+f\left(t, z, z^{\prime}\right)=0, \quad t \neq t_{k}, \\
\left.\Delta z\right|_{t=t_{k}}=0,\left.\quad \Delta z^{\prime}\right|_{t=t_{k}}+f_{k}\left(z, z^{\prime}\right)=0 .
\end{gathered}
$$

Theorem 3.10 (see $[1,10])$. Suppose the following.

(1) The function $f(t, u, v)$ is such that if $z(t)$ is a continuous function for $t \geq \alpha$ having a piecewise continuous derivative $z^{\prime}(t)$ for $t \geq \alpha$, then the function $f\left(t, z(t), z^{\prime}(t)\right)$ is piecewise continuous for $t \geq \alpha$.

(2) The following inequalities are valid:

$$
\begin{aligned}
& u f(t, u, v) \geq q(t) u^{2}, \quad t \geq \alpha,(u, v) \in \mathbb{R}^{2}, \\
& u f_{k}(u, v) \geq q_{k} u^{2}, \quad t_{k} \geq \alpha,(u, v) \in \mathbb{R}^{2} .
\end{aligned}
$$

Then every solution $z(t)$ of (3.17) defined for $t \geq T \geq \alpha$ is oscillatory if (3.7) has an oscillatory solution.

Recently, by establishing a Picone's formula and a Wirtinger type inequality, Özbekler and Zafer [17] have obtained similar results for second-order linear impulsive differential equations of the form

$$
\begin{gathered}
l[x]=\left(k(t) x^{\prime}\right)^{\prime}+r(t) x^{\prime}+p(t) x=0, \quad t \neq \theta_{i}, \\
l_{0}[x]=\Delta\left(k(t) x^{\prime}\right)+p_{i} x=0, \quad t=\theta_{i}, \\
L[y]=\left(m(t) y^{\prime}\right)^{\prime}+s(t) y^{\prime}+q(t) y=0, \quad t \neq \theta_{i}, \\
L_{0}[y]=\Delta\left(m(t) y^{\prime}\right)+q_{i} y=0, \quad t=\theta_{i},
\end{gathered}
$$


where $\left\{p_{i}\right\}$ and $\left\{q_{i}\right\}$ are real sequences, $k, m, r, s, p, q \in \operatorname{PLC}(I)$ with $k(t)>0$ and $m(t)>0$ for all $t \in I \subset J$.

Let $I_{0}$ be a nondegenerate subinterval of $I$. In what follows we shall make use of the following condition:

$$
k(t) \neq m(t) \quad \text { whenever } r(t) \neq s(t) \forall t \in I_{0} .
$$

It is well-known that condition $(\mathrm{H})$ is crucial in obtaining a Picone's formula in the case when impulses are absent. If $(\mathrm{H})$ fails to hold, then Wirtinger, Leighton, and Sturm-Picone type results require employing a so-called "device of Picard." We will show how this is possible for impulsive differential equations as well.

Let $(\mathrm{H})$ be satisfied. Suppose that $x, y \in C\left(I_{0}\right)$ such that $x^{\prime}, y^{\prime} \in \operatorname{PLC}\left(I_{0}\right)$ and $k x^{\prime}, m y^{\prime} \in$ $\operatorname{PLC}^{1}\left(I_{0}\right)$. These conditions simply mean that $x$ and $y$ are in the domain of $l, l_{0}$ and $L, L_{0}$, respectively. If $y(t) \neq 0$ for any $t \in I_{0}$, then we may define

$$
w(t)=\frac{x(t)}{y(t)}\left[y(t) k(t) x^{\prime}(t)-x(t) m(t) y^{\prime}(t)\right] \text { for } t \in I_{0}
$$

For clarity, we suppress the variable $t$. Clearly,

$$
\begin{aligned}
w^{\prime}= & (k-m)\left(x^{\prime}\right)^{2}+(q-p) x^{2}+m\left(x^{\prime}-\frac{x}{y} y^{\prime}\right)^{2}+x^{2} \frac{s y^{\prime}}{y}-r x x^{\prime} \\
& +\frac{x}{y}\{y l[x]-x L[y]\}, \quad t \neq \theta_{i}, \\
\Delta w= & x\left\{l_{0}[x]-p_{i} x\right\}-\frac{x^{2}}{y}\left\{L_{0}[y]-q_{i} y\right\}, \quad t=\theta_{i} .
\end{aligned}
$$

In view of (3.19) and (3.20) it is not difficult to see from (3.22) that

$$
\begin{aligned}
w^{\prime}= & (k-m)\left(x^{\prime}\right)^{2}+(q-p) x^{2}+m\left(x^{\prime}-\frac{x y^{\prime}}{y}\right)^{2}-s x\left(x^{\prime}-\frac{x y^{\prime}}{y}\right) \\
& +(s-r) x x^{\prime}+\frac{x}{y}\{y l[x]-x L[y]\} \\
= & \left\{q-p-\frac{(s-r)^{2}}{4(k-m)}-\frac{s^{2}}{4 m}\right\} x^{2}+(k-m)\left\{x^{\prime}+\frac{(s-r)}{2(k-m)} x\right\}^{2} \\
& +\frac{m}{y^{2}}\left(x^{\prime} y-x y^{\prime}-\frac{s}{2 m} x y\right)^{2}+\frac{x}{y}\{y l[x]-x L[y]\}, \quad t \neq \theta_{i}, \\
\Delta w= & \left(q_{i}-p_{i}\right) x^{2}+\frac{x}{y}\left\{y l_{0}[x]-x L_{0}[y]\right\}, \quad t=\theta_{i} .
\end{aligned}
$$


Employing the identity

$$
w(\beta)-w(\alpha)=\int_{\alpha}^{\beta} w^{\prime}(t) d t+\sum_{\alpha \leq \theta_{i}<\beta} \Delta w\left(\theta_{i}\right),
$$

the following Picone's formula is easily obtained.

Theorem 3.11 (Picone's formula [17]). Let $(\mathrm{H})$ be satisfied. Suppose that $x, y \in C\left(I_{0}\right)$ such that $x^{\prime}, y^{\prime} \in \operatorname{PLC}\left(I_{0}\right)$ and $k x^{\prime}, m y^{\prime} \in \operatorname{PLC}^{1}\left(I_{0}\right)$. If $y(t) \neq 0$ for any $t \in I_{0}$ and $[\alpha, \beta] \subseteq I_{0}$, then

$$
\begin{aligned}
\left.\frac{x}{y}\left(y k x^{\prime}-x m y^{\prime}\right)\right|_{\alpha} ^{\beta}=\int_{\alpha}^{\beta}\{ & {\left[q-p-\frac{(s-r)^{2}}{4(k-m)}-\frac{s^{2}}{4 m}\right] x^{2}+(k-m)\left\{x^{\prime}+\frac{(s-r)}{2(k-m)} x\right\}^{2} } \\
& \left.+\frac{m}{y^{2}}\left(x^{\prime} y-x y^{\prime}-\frac{s}{2 m} x y\right)^{2}+\frac{x}{y}\{y l[x]-x L[y]\}\right\} d t \\
& +\sum_{\alpha \leq \theta_{i}<\beta}\left[\left(q_{i}-p_{i}\right) x^{2}+\frac{x}{y}\left\{y l_{0}[x]-x L_{0}[y]\right\}\right]
\end{aligned}
$$

In a similar manner one may derive a Wirtinger type inequality.

Theorem 3.12 (Wirtinger type inequality [17]). If there exists a solution $x$ of (3.19) such that $x \neq 0$ on $(a, b)$, then

$$
W[\eta]=\int_{a}^{b}\left\{p \eta^{2}-k\left(\eta^{\prime}-\frac{r}{2 k} \eta\right)^{2}\right\} d t+\sum_{a \leq \theta_{i}<b} p_{i} \eta^{2} \leq 0, \quad \eta \in \Omega_{r k}
$$

where

$$
\Omega_{r k}=\left\{\eta \in C[a, b]: r \eta^{\prime} \in \operatorname{PLC}[a, b], k \eta^{\prime} \in \operatorname{PLC}^{1}[a, b], \eta(a)=\eta(b)=0\right\} .
$$

Corollary 3.13. If there exists an $\eta \in \Omega_{r k}$ such that $W[\eta]>0$, then every solution $x$ of (3.19) has a zero in $(a, b)$.

Corollary 3.14. Suppose that for a given $t_{1} \geq t_{0}$ there exists an interval $(a, b) \subset\left[t_{1}, \infty\right)$ and $a$ function $\eta \in \Omega_{r k}$ for which $W[\eta]>0$. Then (3.19) is oscillatory.

Next, we give a Leighton type comparison theorem.

Theorem 3.15 (Leighton type comparison [17]). Suppose that there exists a solution $x \in \Omega_{r k}$ of (3.19). If $(\mathrm{H})$ is satisfied with $(a, b) \subset I_{0}$ and

$$
\begin{aligned}
L[x]:= & \int_{a}^{b}\left\{\left[q-p-\frac{(s-r)^{2}}{4(k-m)}-\frac{s^{2}}{4 m}\right] x^{2}+(k-m)\left[x^{\prime}+\frac{s-r}{2(k-m)} x\right]^{2}\right\} d t \\
& +\sum_{a \leq \theta_{i}<b}\left(q_{i}-p_{i}\right) x^{2}>0,
\end{aligned}
$$

then every solution $y$ of (3.20) must have at least one zero in $(a, b)$. 
Proof. Let $\alpha=a+\epsilon$ and $\beta=b-\epsilon \in I_{0}$. Since $x$ and $y$ are solutions of (3.19) and (3.20), respectively, we have $l[x] \equiv l_{0}[x] \equiv L[y] \equiv L_{0}[y] \equiv 0$. Employing Picone's formula (3.25), we see that

$$
\begin{aligned}
& \left.\frac{x}{y}\left(y k x^{\prime}-x m y^{\prime}\right)\right|_{a+\epsilon} ^{b-\epsilon} \\
& =\int_{a+\epsilon}^{b-\epsilon}\left[\left\{q-p-\frac{(s-r)^{2}}{4(k-m)}-\frac{s^{2}}{4 m}\right\} x^{2}+(k-m)\left\{x^{\prime}+\frac{(s-r)}{2(k-m)} x\right\}^{2}\right. \\
& \left.\quad+\frac{m}{y^{2}}\left\{x^{\prime} y-x y^{\prime}-\frac{s}{2 m} x y\right\}^{2}\right] d t \\
& \quad+\sum_{a+\epsilon \leq \theta_{i}<b-\epsilon}\left(q_{i}-p_{i}\right) x^{2} .
\end{aligned}
$$

The functions under integral sign are all integrable, and regardless of the values of $y(a)$ or $y(b)$, the left-hand side of (3.29) tends to zero as $\epsilon \rightarrow 0^{+}$. Clearly, (3.29) results in

$$
L[x] \leq 0,
$$

which contradicts (3.28).

Corollary 3.16 (Sturm-Picone type comparison). Let $x$ be a solution of (3.19) having two consecutive zeros $a, b \in I_{0}$. Suppose that $(\mathrm{H})$ holds, and

$$
\begin{gathered}
k \geq m, \\
q \geq p+\frac{(s-r)^{2}}{4(k-m)}+\frac{s^{2}}{4 m}
\end{gathered}
$$

for all $t \in[a, b]$, and

$$
q_{i} \geq p_{i}
$$

for all $i \in \mathbb{N}$ for which $\theta_{i} \in[a, b]$.

If either (3.31) or (3.32) is strict in a subinterval of $[a, b]$ or (3.33) is strict for some $i \in \mathbb{N}$, then every solution $y$ of (3.20) must have at least one zero on $(a, b)$.

Corollary 3.17. Suppose that conditions (3.31)-(3.32) are satisfied for all $t \in\left[t_{*}, \infty\right)$ for some integer $t_{*} \geq t_{0}$, and that (3.33) is satisfied for all $i \in \mathbb{N}$ for which $\theta_{i} \geq t_{*}$. If one of the inequalities (3.31)-(3.33) is strict, then (3.20) is oscillatory whenever any solution $x$ of (3.19) is oscillatory.

As a consequence of Theorem 3.15 and Corollary 3.16, we have the following oscillation result.

Corollary 3.18. Suppose for a given $t_{1} \geq t_{0}$ there exists an interval $(a, b) \subset\left[t_{1}, \infty\right)$ for which that condition of either Theorem 3.15 or Corollary 3.16 are satisfied. Then (3.20) is oscillatory. 
If $(\mathrm{H})$ does not hold, we introduce a setting, which is based on a device of Picard, leading to different versions of Corollary 3.16.

Indeed, for any $h \in \operatorname{PLC}^{1}(I)$, we have

$$
\frac{d}{d t}\left(x^{2} h\right)=2 x x^{\prime} h+x^{2} h^{\prime}, \quad t \neq \theta_{i}
$$

Let

$$
v:=\frac{x}{y}\left(y k x^{\prime}-x m y^{\prime}\right)+x^{2} h, \quad t \in I
$$

It follows that

$$
\begin{aligned}
v^{\prime}= & \left\{q-p+h^{\prime}-\frac{(s-r+2 h)^{2}}{4(k-m)}-\frac{s^{2}}{4 m}\right\} x^{2}+(k-m)\left\{x^{\prime}+\frac{s-r+2 h}{2(k-m)} x\right\}^{2} \\
& +\frac{m}{y^{2}}\left\{x^{\prime} y-x y^{\prime}-\frac{s}{2 m} x y\right\}^{2}, \quad t \neq \theta_{i}, \\
\Delta v= & \left(q_{i}-p_{i}\right) x^{2}+x^{2} \Delta h, \quad t=\theta_{i} .
\end{aligned}
$$

Assuming that $r, s \in \operatorname{PLC}^{1}(I)$, the choice of $h=(r-s) / 2$ yields

$$
\begin{gathered}
v^{\prime}=\left\{q-p-\frac{s^{\prime}-r^{\prime}}{2}-\frac{s^{2}}{4 m}\right\} x^{2}+(k-m)\left(x^{\prime}\right)^{2}+\frac{m}{y^{2}}\left\{x^{\prime} y-x y^{\prime}-\frac{s}{2 m} x y\right\}^{2}, \quad t \neq \theta_{i}, \\
\Delta v=\left\{q_{i}-p_{i}-\frac{1}{2}(\Delta s-\Delta r)\right\} x^{2}, \quad t=\theta_{i} .
\end{gathered}
$$

Then, we have the following result.

Theorem 3.19 (Device of Picard [17]). Let $r, s \in P L C^{1}(I)$ and let $x$ be a solution of (3.19) having two consecutive zeros $a$ and $b$ in I. Suppose that

$$
\begin{gathered}
k \geq m, \\
q \geq p+\frac{1}{2}\left(s^{\prime}-r^{\prime}\right)+\frac{s^{2}}{4 m}
\end{gathered}
$$

are satisfied for all $t \in[a, b]$, and that

$$
q_{i} \geq p_{i}+\frac{1}{2}(\Delta s-\Delta r), \quad t=\theta_{i}
$$

for all $i \in \mathbb{N}$ for which $\theta_{i} \in[a, b]$.

If either (3.38) or (3.39) is strict in a subinterval of $[a, b]$ or (3.40) is strict for some $i$, then any solution $y$ of (3.20) must have at least one zero in $(a, b)$. 
Corollary 3.20. Suppose that (3.38)-(3.39) are satisfied for all $t \in\left[t_{*}, \infty\right)$ for some integer $t_{*} \geq t_{0}$, and that (3.40) is satisfied for all $i \in \mathbb{N}$ for which $\theta_{i} \geq t_{*}$. If $r, s \in P L C^{1}\left[t_{*}, \infty\right)$ and one of the inequalities (3.38)-(3.40) is strict, then (3.20) is oscillatory whenever any solution $x$ of (3.19) is oscillatory.

As a consequence of Theorem 3.19, we have the following Leighton type comparison result which is analogous to Theorem 3.15.

Theorem 3.21 (Leighton type comparison [17]). Let $r, s \in P L C^{1}[a, b]$. If there exists a solution $x \in \Omega_{r k}$ of (3.19) such that

$$
\begin{aligned}
\mathcal{L}[x]:= & \int_{a}^{b}\left\{\left[q-p-\frac{1}{2}\left(s^{\prime}-r^{\prime}\right)-\frac{s^{2}}{4 m}\right] x^{2} d t+(k-m)\left(x^{\prime}\right)^{2}\right\} d t \\
& +\sum_{a \leq \theta_{i}<b}\left\{q_{i}-p_{i}-\frac{1}{2}(\Delta s-\Delta r)\right\} x^{2}>0,
\end{aligned}
$$

then every solution $y$ of (3.20) must have at least one zero in $(a, b)$.

As a consequence of Theorems 3.19 and 3.21, we have the following oscillation result.

Corollary 3.22. Suppose that for a given $t_{1} \geq t_{0}$ there exists an interval $(a, b) \subset\left[t_{1}, \infty\right)$ for which conditions of either Theorem 3.19 or Theorem 3.21 are satisfied. Then (3.20) is oscillatory.

Moreover, it is possible to obtain results for (3.20) analogous to Theorem 3.12 and Corollary 3.13.

Theorem 3.23 (Wirtinger type inequality [17]). If there exists a solution $y$ of (3.20) such that $y \neq 0$ on $(a, b)$, then for $s \in P L C^{1}[a, b]$ and for all $\eta \in \Omega_{\text {sm }}$,

$$
\mathcal{W}[\eta]:=\int_{a}^{b}\left\{\left(q-\frac{s^{2}}{2 m}-\frac{s^{\prime}}{2}\right) \eta^{2}-m\left(\eta^{\prime}\right)^{2}\right\} d t+\sum_{a \leq \theta_{i}<b}\left(q_{i}-\frac{1}{2} \Delta s\right) \eta^{2} \leq 0 .
$$

Corollary 3.24. If there exists an $\eta \in \Omega_{s m}$ with $s \in P L C^{1}[a, b]$ such that $\mathcal{W}[\eta]>0$, then every solution $y$ of (3.20) must have at least one zero in $(a, b)$.

As an immediate consequence of Corollary 3.24, we have the following oscillation result.

Corollary 3.25. Suppose that for a given $t_{1} \geq t_{0}$ there exists an interval $(a, b) \subset\left[t_{1}, \infty\right)$ and $a$ function $\eta \in \Omega_{s m}$ with $s \in P L C^{1}(I)$ for which $\mathcal{W}[\eta]>0$. Then (3.20) is oscillatory.

\subsection{Oscillation of Second-Order Linear Equations}

The oscillation theory of second-order impulsive differential equations has developed rapidly in the last decade. For linear equations, we refer to the papers [11, 19-21]. 
Let us consider the second-order linear differential equation with impulses

$$
\begin{gathered}
x^{\prime \prime}+p(t) x=0, \quad t \neq t_{k}, \\
x\left(t_{k}^{+}\right)=b_{k} x\left(t_{k}\right), \quad x^{\prime}\left(t_{k}^{+}\right)=c_{k} x^{\prime}\left(t_{k}\right), \quad k \in \mathbb{N},
\end{gathered}
$$

where $p \in C(J, \mathbb{R}),\left\{b_{k}\right\}$ and $\left\{c_{k}\right\}$ are two known sequences of real numbers, and

$$
x^{\prime}\left(t_{k}\right)=\lim _{h \rightarrow 0^{-}} \frac{x\left(t_{k}+h\right)-x\left(t_{k}\right)}{h}, \quad x^{\prime}\left(t_{k}^{+}\right)=\lim _{h \rightarrow 0^{+}} \frac{x\left(t_{k}+h\right)-x\left(t_{k}^{+}\right)}{h} .
$$

For (3.43), it is clear that if $b_{k} \leq 0$ for all large $k$, then (3.43) is oscillatory. So, we assume that $b_{k}>0, k=1,2, \ldots$. The following theorem gives the relation between the existence of oscillatory solutions of (3.43) and the existence of oscillatory solutions of second-order linear nonimpulsive differential equation:

$$
y^{\prime \prime}+p(t) y=0
$$

Theorem 3.26 (see [19]). Assume that $b_{k}=c_{k}, k=1,2, \ldots$ Then the oscillation of all solutions of (3.43) is equivalent to the oscillation of all solutions of (3.45).

Proof. Let $x(t)$ be any solution of (3.43). Set $y(t)=x(t) \prod_{t_{0} \leq t_{k}<t} b_{k}^{-1}$ for $t>t_{0}$. Then, for all $n \geq 1$, we have

$$
y\left(t_{n}^{+}\right)=x\left(t_{n}\right) b_{n} \prod_{t_{0} \leq t_{k} \leq t_{n}} b_{k}^{-1}=x\left(t_{n}\right) \prod_{t_{0} \leq t_{k}<t_{n}} b_{k}^{-1}=y\left(t_{n}\right) .
$$

Thus, $y(t)$ is continuous on $J$. Furthermore, for $t \neq t_{n}, n \in \mathbb{N}$, we have

$$
y^{\prime}(t)=\left(\prod_{t_{0} \leq t_{k}<t} b_{k}^{-1}\right) \lim _{h \rightarrow 0} \frac{x(t+h)-x(t)}{h}=x^{\prime}(t) \prod_{t_{0} \leq t_{k}<t} b_{k}^{-1} .
$$

For $t=t_{n}$, it can be shown that

$$
y^{\prime}\left(t_{n}^{-}\right)=y^{\prime}\left(t_{n}^{+}\right)=x^{\prime}\left(t_{n}\right) \prod_{t_{0} \leq t_{k}<t_{n}} b_{k}^{-1} .
$$

Thus, $y^{\prime}(t)$ is continuous if we define the value of $y^{\prime}(t)$ at $t=t_{n}$ as

$$
y^{\prime}\left(t_{n}\right)=x^{\prime}\left(t_{n}\right) \prod_{t_{0} \leq t_{k}<t_{n}} b_{k}^{-1} .
$$

Now, we have for $t \neq t_{n}$,

$$
y^{\prime \prime}(t)=x^{\prime \prime}(t) \prod_{t_{0} \leq t_{k}<t} b_{k}^{-1}=-p(t) y(t)
$$


and for $t=t_{n}$

$$
y^{\prime \prime}\left(t_{n}^{-}\right)=y^{\prime \prime}\left(t_{n}^{+}\right)=-p\left(t_{n}\right) y\left(t_{n}\right)
$$

Thus, we obtain

$$
y^{\prime \prime}(t)+p(t) y(t)=0, \quad t \geq t_{0}
$$

This shows that $y(t)$ is the solution of (3.45).

Conversely, if $y(t)$ is the continuous solution of (3.45), we set $x(t)=y(t) \prod_{t_{0} \leq t_{k}<t} b_{k}$ for $t>t_{0}$. Then, $x\left(t_{n}^{-}\right)=x\left(t_{n}\right)$, and $x\left(t_{n}^{+}\right)=y\left(t_{n}\right) \prod_{t_{0} \leq t_{k} \leq t_{n}} b_{k}=b_{n} x\left(t_{n}\right)$. Furthermore, for $t \neq t_{n}$, we have

$$
x^{\prime}(t)=y^{\prime}(t) \prod_{t_{0} \leq t_{k}<t} b_{k}, \quad x^{\prime \prime}(t)=y^{\prime \prime}(t) \prod_{t_{0} \leq t_{k}<t} b_{k}
$$

and so

$$
\begin{gathered}
x^{\prime}\left(t_{n}^{+}\right)=b_{n} y^{\prime}\left(t_{n}^{-}\right) \prod_{t_{0} \leq t_{k}<t_{n}} b_{k}=c_{n} x^{\prime}\left(t_{n}\right), \\
x^{\prime \prime}(t)+p(t) x(t)=\left(\prod_{t_{0} \leq t_{k}<t} b_{k}\right)\left[y^{\prime \prime}(t)+p(t) y(t)\right]=0, \quad t \neq t_{n} .
\end{gathered}
$$

Thus, $x(t)$ is the solution of (3.43). This completes the proof.

By Theorems 3.26 and 2.3, one may easily get the following corollary.

Corollary 3.27. Assume that $p(t) \geq 0, b_{k}=c_{k}, k \in \mathbb{N}$. Then, (3.43) is oscillatory if

$$
\liminf _{t \rightarrow \infty} t \int_{t}^{\infty} p(s) d s>\frac{1}{4}
$$

and nonoscillatory if

$$
t \int_{t}^{\infty} p(s) d s \leq \frac{1}{4}, \text { for large } t
$$

When $b_{k} \neq c_{k}$, and $b_{k}, c_{k}>0, k \in \mathbb{N}$, oscillation criteria for (3.43) can be obtained by means of a Riccati technique as well. First, we need the following lemma.

Lemma 3.28. Assume that $p(t) \geq 0, p(t) \not \equiv 0$ on any interval $\left(t_{k}, t_{k+1}\right]$, and let $x(t)$ be an eventually positive solution of (3.43). If

$$
\sum_{n=0}^{\infty}\left(\prod_{i=0}^{n} \frac{c_{i}}{b_{i}}\right)\left(t_{n+1}-t_{n}\right)=\infty,
$$

where $b_{0}=c_{0}=1$, then, eventually $x^{\prime}(t)>0$. 
Now, let $x(t)$ be an eventually positive solution of (3.43) such that $x(t)>0$ and $x^{\prime}(t)>0$ for $t \geq T \geq t_{0}$. Under conditions of Lemma 3.28, let $u(t)=x^{\prime}(t) / x(t)$, for $t \geq T$. Then, (3.43) leads to an impulsive Riccati equation:

$$
\begin{gathered}
u^{\prime}(t)+u^{2}(t)+p(t)=0, \quad t \geq T, \quad t \neq t_{k} \\
u\left(t_{k}^{+}\right)=d_{k} u\left(t_{k}\right), \quad t_{k} \geq T
\end{gathered}
$$

where $d_{k}=c_{k} / b_{k}, k \in \mathbb{N}$.

Theorem 3.29 (see [19]). Equation (3.43) is oscillatory if the second-order self-adjoint differential equation

$$
\left[\left(\prod_{T \leq t_{k}<t} d_{k}^{-1}\right) y^{\prime}(t)\right]^{\prime}+p(t)\left(\prod_{T \leq t_{k}<t} d_{k}^{-1}\right) y(t)=0, \quad t>T \geq t_{0}
$$

is oscillatory, where $d_{k}=c_{k} / b_{k}, k \in \mathbb{N}$.

Proof. Assume, for the sake of contradiction, that (3.43) has a nonoscillatory solution $x(t)$, such that $x(t)>0$, for $t \geq T \geq t_{0}$. Now, define

$$
v(t)=\left(\prod_{T \leq t_{k}<t} d_{k}^{-1}\right) u(t), \quad t>T
$$

Then, it can be shown that $v(t)$ is continuous and satisfies

$$
v^{\prime}(t)+\left(\prod_{T \leq t_{k}<t} d_{k}\right) v^{2}(t)+\left(\prod_{T \leq t_{k}<t} d_{k}^{-1}\right) p(t)=0, \quad t>T .
$$

Next, we define

$$
w(t)=\exp \left[\int_{T}^{t}\left(\prod_{T \leq t_{k}<s} d_{k}\right) v(s) d s\right], \quad t>T
$$

Then $w(t)>0$ is a solution of (3.59). This completes the proof.

By Theorems 3.29 and 2.4, we have the following corollary.

Corollary 3.30. Assume that

$$
\int_{T}^{\infty}\left(\prod_{T \leq t_{k}<t} d_{k}\right) d t=\infty, \quad \int_{T}^{\infty}\left(\prod_{T \leq t_{k}<t} d_{k}^{-1}\right) p(t) d t=\infty,
$$

where $d_{k}=c_{k} / b_{k}, k \in \mathbb{N}$. Then, (3.43) is oscillatory. 
Example 3.31 (see [19]). Consider the equation

$$
\begin{gathered}
x^{\prime \prime}(t)+\frac{1}{4 t^{2}} x(t)=0, \quad t \geq \frac{1}{2}, t \neq k, \\
x\left(k^{+}\right)=x(k), \quad x^{\prime}\left(k^{+}\right)=\frac{k}{k+1} x^{\prime}(k), \quad k \in \mathbb{N} .
\end{gathered}
$$

If $T \in(m, m+1]$ for some integer $m \geq 0$, then it is easy to see that

$$
\int_{T}^{\infty}\left(\prod_{T \leq t_{k}<t} d_{k}\right) d t \geq \int_{T}^{\infty}\left(\prod_{T \leq k<[t]+1} \frac{k}{k+1}\right) d t=\int_{T}^{\infty} \frac{m+1}{[t]+1} d t=\infty,
$$

where $[\cdot]$ denotes the greatest integer function, and

$$
\int_{T}^{\infty}\left(\prod_{T \leq t_{k}<t} d_{k}^{-1}\right) p(t) d t \geq \int_{T}^{\infty}\left(\prod_{T \leq k<[t]} \frac{k+1}{k}\right) \frac{1}{4 t^{2}} d t=\frac{1}{m+1} \int_{T}^{\infty} \frac{[t]}{4 t^{2}} d t=\infty
$$

Thus, by Corollary 3.30, (3.64) is oscillatory. We note that the corresponding differential equation without impulses

$$
y^{\prime \prime}+\frac{1}{4 t^{2}} y=0
$$

is nonoscillatory by Theorem 2.3.

In [20], Luo and Shen used the above method to discuss the oscillation and nonoscillation of the second-order differential equation:

$$
\begin{gathered}
\left(a(t) x^{\prime}\right)^{\prime}+p(t) x=0, \quad t \neq t_{k}, \\
x\left(t_{k}^{+}\right)=b_{k} x\left(t_{k}\right), \quad x^{\prime}\left(t_{k}^{+}\right)=c_{k} x^{\prime}\left(t_{k}\right), \quad k \in \mathbb{N},
\end{gathered}
$$

where $a \in C(J,(0, \infty)), p \in C(J, \mathbb{R})$.

In [21], the oscillatory and nonoscillatory properties of the second-order linear impulsive differential equation

$$
u^{\prime \prime}=-p(t) u, \quad t \geq 0
$$

is investigated, where

$$
p(t)=\sum_{n=1}^{\infty} a_{n} \delta\left(t-t_{n}\right)
$$


$a_{n}>0$ for all $n \in \mathbb{N}$, and $\delta(t)$ is the $\delta$-function, that is,

$$
\int_{-\infty}^{\infty} \delta(t) \varphi(t) d t=\int_{-\varepsilon}^{\varepsilon} \delta(t) \varphi(t) d t=\varphi(0)
$$

for all $\varphi(t)$ being continuous at $t=0$. Before giving the main result, we need the following lemmas. For each $n \in \mathbb{N}$, define the sequence $\left\{P_{k}^{n}\right\}_{k=1}^{\infty}$ inductively by

$$
\begin{gathered}
P_{1}^{n}=a_{n+1}\left(t_{n+1}-t_{n}\right), \\
P_{k+1}^{n}=\frac{a_{n+k+1}}{a_{n+k}}\left(\frac{P_{k}^{n}}{1-P_{k}^{n}}+a_{n+k}\left(t_{n+k+1}-t_{n+k}\right)\right),
\end{gathered}
$$

where $P_{k+1}^{n}=\infty$ provided $P_{k}^{n}=1$ and $P_{k+1}^{n}=0$ provided $P_{k}^{n}=\infty$. Let $S_{n}=\sup \left\{P_{k}^{n}: k \in \mathbb{N}\right\}$.

Lemma 3.32. If $S_{n_{0}} \leq 1$ for some $n_{0} \in N$, then $S_{n_{0}+1} \leq 1$, and $S_{n} \leq 1$ for all $n \geq n_{0}$.

Proof. By induction and in view of the fact that the function $f(x)=x /(1-x)$ is increasing in $x \in[0,1)$, it can be seen that

$$
0<P_{k}^{n_{0}+1} \leq P_{k+1}^{n_{0}}<1, \quad \forall k \in N
$$

Hence, $S_{n_{0}+1} \leq S_{n_{0}} \leq 1$.

The next lemma can also be proved by induction.

Lemma 3.33. Suppose that $0<\alpha_{k} \leq \beta_{k}$ and $\lambda_{k}>0$ for all $k \in N$. Define, by induction,

$$
\begin{gathered}
p_{1}=\alpha_{1} \lambda_{1}, \quad q_{1}=\beta_{1} \lambda_{1}, \\
p_{k+1}=\frac{\alpha_{k+1}}{\alpha_{k}}\left(\frac{p_{k}}{1-p_{k}}+\alpha_{k} \lambda_{k+1}\right), \\
q_{k+1}=\frac{\beta_{k+1}}{\beta_{k}}\left(\frac{q_{k}}{1-q_{k}}+\beta_{k} \lambda_{k+1}\right), \quad k \in \mathbb{N} .
\end{gathered}
$$

If $0<q_{k}<1$ for all $k \in N$, then

$$
0<p_{k} \leq \frac{\alpha_{k}}{\beta_{k}} q_{k}<1, \quad \forall k \in N
$$

The following theorem is the main result of [21]. The proof uses the above two lemmas and the induction principle.

Theorem 3.34. The following statements are equivalent.

(i) There is $n_{0} \in N$ such that $S_{n_{0}} \leq 1$. 
(ii) There is $n_{0} \in N$ such that $S_{n} \leq 1$ for all $n \geq n_{0}$.

(iii) Equation (3.69) is nonoscillatory.

(iv) Equation (3.69) has a nonoscillatory solution.

Applying Theorem 3.34, the nonoscillation and oscillation of (3.69), in the case of $t_{n}=$ $t_{0}+\lambda^{n-1} T, \lambda>1, T>0$, and $t_{n}=t_{0}+n T$, are investigated in [21].

In all the publications mentioned above, the authors have considered differential equations with fixed moments of impulse actions. That is, it is assumed that the jumps happen at fixed points. However, jumps can be at random points as well. The oscillation of impulsive differential equations with random impulses was investigated in [11]. Below we give the results obtained in this case.

Let $\tau_{k}$ be a random variable defined in $D_{k} \equiv\left(0, d_{k}\right), k \in \mathbb{N}, 0<d_{k} \leq \infty$, and let $\tau \in \mathbb{R}$ be a constant. Consider the second-order linear differential equation with random impulses:

$$
\begin{gathered}
y^{\prime \prime}+a(t) y^{\prime}+p(t) y=0, \quad t \in R_{\tau}, t \neq \xi_{k}, \\
\Delta y\left(\xi_{k}\right)=b_{k}\left(\tau_{k}\right) y\left(\xi_{k}^{-}\right), \quad \Delta y^{\prime}\left(\xi_{k}\right)=b_{k}\left(\tau_{k}\right) y^{\prime}\left(\xi_{k}^{-}\right), \quad k \in \mathbb{N},
\end{gathered}
$$

where $R_{\tau}=[\tau, \infty), a, p: R_{\tau} \rightarrow \mathbb{R}$ are Lebesque measurable and locally essentially bounded functions, $\xi_{0}=t_{0} \in R_{\tau}, \xi_{k}=\xi_{k-1}+\tau_{k}$ for all $k \in \mathbb{N}, \Delta y\left(\xi_{k}\right)=y\left(\xi_{k}\right)-y\left(\xi_{k}^{-}\right), \Delta y^{\prime}\left(\xi_{k}\right)=$ $y^{\prime}\left(\xi_{k}\right)-y^{\prime}\left(\xi_{k}^{-}\right)$, and $y\left(\xi_{k}^{-}\right)=\lim _{t \rightarrow \xi_{k}-0} y(t)$.

Definition 3.35. Let $X$ be a real-valued random variable in the probability space $(\Omega, F, P)$, where $\Omega$ is the sample space, $F$ is the $\sigma$-field, and $P(\cdot)$ is the probability measure. If $\int_{\Omega}|x| P(d x)<\infty$, then $\int_{\Omega} x P(d x)$ is called the expectation of $X$ and is denoted by $E(X)$, that is,

$$
E(X)=\int_{\Omega} x P(d x)
$$

In particular, if $X$ is a continuous random variable having probability density function $f(x)$, then

$$
E(X)=\int_{-\infty}^{\infty} x f(x) d x
$$

Definition 3.36. A stochastic process $y(t)$ is said to be a sample path solution to (3.76) with the initial condition $y_{t_{0}}=y_{0}$ if for any sample value $t_{1}<t_{2}<\cdots<t_{k}<\cdots$ of $\left\{\xi_{k}\right\}_{k \geq 1}$, then $y(t)$ satisfies

$$
\begin{aligned}
& y^{\prime \prime}+a(t) y^{\prime}+p(t) y=0, \quad t \in R_{\tau}, t \neq t_{k}, \\
& \Delta y\left(t_{k}\right)=b_{k}\left(t_{k}-t_{k-1}\right) y\left(t_{k}^{-}\right), \forall k \in \mathbb{N}, \\
& \Delta y^{\prime}\left(t_{k}\right)=b_{k}\left(t_{k}-t_{k-1}\right) y^{\prime}\left(t_{k}^{-}\right), \quad \forall k \in \mathbb{N} .
\end{aligned}
$$


Definition 3.37. The exponential distribution is a continuous random variable with the probability density function:

$$
f(x)= \begin{cases}\lambda e^{-\lambda x}, & x>0 \\ 0, & \text { elsewhere }\end{cases}
$$

where $\lambda>0$ is a parameter.

Definition 3.38. A solution $y(t)$ of (3.76) is said to be nonoscillatory in mean if $E[y(t)]$ is either eventually positive or eventually negative. Otherwise, it is called oscillatory.

Consider the following auxiliary differential equation:

$$
x^{\prime \prime}+a(t) x^{\prime}+p(t) x=0, \quad t \in R_{\tau} .
$$

Lemma 3.39. The function $y(t)$ is a solution of (3.76) if and only if

$$
y(t)=\sum_{k=0}^{\infty}\left(\prod_{i=1}^{k}\left[1+b_{i}\left(\tau_{i}\right)\right]_{\left[\tilde{L}_{k}, \tilde{\xi}_{k+1}\right)}(t)\right) x(t),
$$

where $x(t)$ is a solution of (3.81) with the same initial conditions for (3.76), and $x$ is the index function, that is,

$$
X_{\left[\tilde{\xi}_{k}, \tilde{\xi}_{k+1}\right)}(t)= \begin{cases}1, & \text { if } \xi_{k} \leq t<\xi_{k+1} \\ 0, & \text { otherwise. }\end{cases}
$$

Proof. If $x(t)$ is a solution of system (3.81), for any $t_{0}<t_{1}<\cdots<t_{k}<\cdots$, we have

$$
y(t)=\sum_{k=0}^{\infty}\left(\prod_{i=1}^{k}\left[1+b_{i}\left(t_{i}-t_{i-1}\right)\right] \chi_{\left[t_{k}, t_{k+1}\right)}(t)\right) x(t) .
$$

It can be seen that

$$
\begin{gathered}
y^{\prime \prime}(t)+a(t) y^{\prime}(t)+p(t) y(t)=0, \quad t \neq t_{k} \\
y\left(t_{k}\right)=\left[1+b_{k}\left(t_{k}-t_{k-1}\right)\right] y\left(t_{k}^{-}\right), \quad y^{\prime}\left(t_{k}\right)=\left[1+b_{k}\left(t_{k}-t_{k-1}\right)\right] y^{\prime}\left(t_{k}^{-}\right),
\end{gathered}
$$

which imply that $y(t)$ satisfies (3.76), that is, $y(t)$ is a sample path solution of (3.76). If $y(t)$ is a sample path solution of (3.76), then it is easy to check that $x(t)$ is a solution of (3.81). This completes the proof.

Theorem 3.40 (see [11]). Let the following condition hold.

(C) Let $\tau_{k}$ be exponential distribution with parameter $\lambda>0, k \in \mathbb{N}$, and let $\tau_{i}$ be independent of $\tau_{j}$ if $i \neq j$. 
Advances in Difference Equations

If there exists $T \in R_{\tau}$ such that

$$
\sum_{k=0}^{\infty} \lambda^{k} \underbrace{\int \cdots \int}_{v_{1}+\cdots+v_{k} \leq t-t_{0}} \prod_{i=1}^{k}\left[1+b_{i}\left(v_{i}\right)\right] d v_{1} \cdots d v_{k}
$$

does not change sign for all $t \geq T$, then all solutions of (3.76) are oscillatory in mean if and only if all solutions of (3.81) are oscillatory.

Proof. Let $y(t)$ be any sample path solution of (3.76); then Lemma 3.39 implies

$$
y(t)=\sum_{k=0}^{\infty}\left(\prod_{i=1}^{k}\left[1+b_{i}\left(\tau_{i}\right)\right]_{\left[\check{\xi}_{k}, \check{\xi}_{k+1}\right)}(t)\right) x(t),
$$

where $x(t)$ is a solution of (3.81). Hence,

$$
\begin{aligned}
E[y(t)] & =E\left[\sum_{k=0}^{\infty}\left(\prod_{i=1}^{k}\left[1+b_{i}\left(\tau_{i}\right)\right] \chi_{\left[\tilde{\xi}_{k}, \tilde{\xi}_{k+1}\right)}(t)\right) x(t)\right] \\
& =\sum_{k=0}^{\infty} E\left(\prod_{i=1}^{k}\left[1+b_{i}\left(\tau_{i}\right)\right]_{\left[\tilde{\xi}_{k}, \tilde{\xi}_{k+1}\right)}(t)\right) x(t) .
\end{aligned}
$$

Further, it can be seen that

$$
\begin{aligned}
& E\left(\prod_{i=1}^{k}\left[1+b_{i}\left(\tau_{i}\right)\right] \chi_{\left[\xi_{k}, \xi_{k+1}\right)}(t)\right) \\
& \quad=\lambda^{k+1} \underbrace{\int \cdots \int}_{\sum_{i=1}^{k} \underbrace{}_{i} \leq t-t_{0}<\sum_{i=1}^{k+1}} \prod_{i=1}^{k}\left[1+b_{i}\left(v_{i}\right)\right] e^{-\lambda v_{1}} \cdots e^{-\lambda v_{k}} e^{-\lambda v_{k+1}} d v_{1} \cdots d v_{k+1} \\
& =\lambda^{k} e^{-\lambda\left(t-t_{0}\right)} \underbrace{\int \cdots \int}_{v_{1}+\cdots+v_{k} \leq t-t_{0}} \prod_{i=1}^{k}\left[1+b_{i}\left(v_{i}\right)\right] d v_{1} \cdots d v_{k} .
\end{aligned}
$$

So,

$$
E[y(t)]=e^{-\lambda\left(t-t_{0}\right)} x(t) \sum_{k=0}^{\infty} \lambda^{k} \underbrace{\int \cdots \int}_{v_{1}+\cdots+v_{k} \leq t-t_{0}} \prod_{i=1}^{k}\left[1+b_{i}\left(v_{i}\right)\right] d v_{1} \cdots d v_{k} .
$$

By assumption, $E[y(t)]$ has the same sign as $x(t)$ for all $t \geq T$. That is, all solutions of (3.76) are oscillatory in mean if and only if all solutions of (3.81) are oscillatory. This completes the proof. 
When $b_{k}\left(\tau_{k}\right)=b_{k}$ is finite, $k \in \mathbb{N}$, then the following result can be proved.

Theorem 3.41 (see [11]). Let condition (C) hold, and let $b_{k}\left(\tau_{k}\right) \equiv b_{k}$ be finite for all $k \in \mathbb{N}$. Further assume that there are a finite number of $b_{k}$ such that $b_{k}<-1$. Then all solutions of (3.76) are oscillatory in mean if and only if all solutions of (3.81) are oscillatory.

\subsection{Oscillation of Higher-Order Linear Equations}

Unlike the second-order impulsive differential equations, there are only very few papers on the oscillation of higher-order linear impulsive differential equations. Below we provide some results for third-order equations given in [22]. For higher-order liner impulsive differential equations we refer to the papers [23, 24].

Let us consider the third-order linear impulsive differential equation of the form

$$
\begin{gathered}
x^{\prime \prime \prime}+p(t) x=0, \quad t \neq t_{k}, \\
x\left(t_{k}^{+}\right)=a_{k} x\left(t_{k}\right), \quad x^{\prime}\left(t_{k}^{+}\right)=b_{k} x^{\prime}\left(t_{k}\right), \quad x^{\prime \prime}\left(t_{k}^{+}\right)=c_{k} x^{\prime \prime}\left(t_{k}\right), \quad k \in \mathbb{N}, \\
x\left(t_{0}^{+}\right)=x_{0}, \quad x^{\prime}\left(t_{0}^{+}\right)=x_{0}^{\prime}, \quad x^{\prime \prime}\left(t_{0}^{+}\right)=x_{0}^{\prime \prime},
\end{gathered}
$$

where $a_{k}>0, b_{k}>0, c_{k}>0, t_{k+1} \geq t_{k}(k \in \mathbb{N})$ and $p \in C\left(J, \mathbb{R}^{+}\right)$is not always zero in $\left(t_{k}, t_{k+1}\right](k \in \mathbb{N})$ for sufficiently large $k$.

The following lemma is a generalization of Lemma 1 in [25].

Lemma 3.42 (see [22]). Assume that $x(t)$ is a solution of (3.91) and there exists $T \geq t_{0}$ such that for any $t \geq T, x(t)>0(<0)$. Let the following conditions be fulfilled.

(C1) One has

$$
\left(t_{1}-t_{0}\right)+\frac{c_{1}}{b_{1}}\left(t_{2}-t_{1}\right)+\cdots+\frac{c_{1} \cdots c_{n}}{b_{1} \cdots b_{n}}\left(t_{n+1}-t_{n}\right)+\cdots=\infty
$$

(C2) One has

$$
\left(t_{1}-t_{0}\right)+\frac{b_{1}}{a_{1}}\left(t_{2}-t_{1}\right)+\cdots+\frac{b_{1} \cdots b_{n}}{a_{1} \cdots a_{n}}\left(t_{n+1}-t_{n}\right)+\cdots=\infty .
$$

Then for sufficiently large teither $(A)$ or $(B)$ holds, where

(A) one has

$$
x^{\prime \prime}(t)>0(<0), \quad x^{\prime}(t)>0(<0) ;
$$

(B) one has

$$
x^{\prime \prime}(t)>0(<0), \quad x^{\prime}(t)<0(>0) .
$$


Theorem 3.43 (see [22]). Assume that conditions of Lemma 3.42 are fulfilled and for any $n \in$ $\mathbb{N}, a_{n}>0,1 \geq b_{n}>0$, and $1 \geq c_{n}>0$. Moreover, assume that the sequence of numbers $\left\{\prod_{i=1}^{n} a_{i}\right\}$ has a positive lower bound, $\sum_{n=1}^{\infty}\left|a_{n}-1\right|$ converges, and $\int^{\infty} t^{2} p(t) d t=\infty$ holds. Then every bounded solution of (3.91) either oscillates or tends asymptotically to zero with fixed sign.

Proof. Suppose that $x(t)$ is a bounded nonoscillatory solution of (3.91) and $x(t)>0, t \geq T \geq$ $t_{0}$. According to Lemma 3.42, either $(A)$ or $(B)$ is satisfied. We claim that $(A)$ does not hold. Otherwise, $x^{\prime}\left(t_{k}\right)>0$ for some $t_{k} \geq T_{0}$. Since $x^{\prime \prime}(t)>0$, it follows that $x^{\prime}(t)$ is monotonically increasing for $t \in\left(t_{k+i-1}, t_{k+i}\right], i \in \mathbb{N}$. For any $t \in\left(t_{k}, t_{k+1}\right], x^{\prime}(t)>x^{\prime}\left(t_{k}^{+}\right)=\gamma>0$. By induction, it can be seen that

$$
x^{\prime}(t)>b_{k+n-1} \cdots b_{k+1} \gamma>0,
$$

in particular,

$$
\begin{gathered}
x^{\prime}\left(t_{k+n}\right)>b_{k+n-1} \cdots b_{k+1} \gamma>0 \\
x^{\prime}\left(t_{k+n}^{+}\right)=b_{k+n} x\left(t_{k+n}\right)>b_{k+n} \cdots b_{k+1} \gamma>0 .
\end{gathered}
$$

Integrating $x^{\prime}(t)>\gamma$ from $t_{k}$ to $t_{k+1}$, we obtain

$$
x\left(t_{k+1}\right) \geq x\left(t_{k}^{+}\right)+\gamma\left(t_{k+1}-t_{k}\right) .
$$

By induction, for any natural number $n \geq 2$, we have

$$
\begin{array}{r}
x\left(t_{k+n}\right) \geq a_{k+n-1} \cdots a_{k+1}[ \\
x^{\prime}\left(t_{k}^{+}\right)+\gamma\left(t_{k+1}-t_{k}\right)+\gamma \frac{b_{k+1}}{a_{k+1}}\left(t_{k+2}-t_{k+1}\right) \\
\left.+\cdots+\gamma \frac{b_{k+n-1} \cdots b_{k+1}}{a_{k+n-1} \cdots a_{k+1}}\left(t_{k+n}-t_{k+n-1}\right)\right] .
\end{array}
$$

Considering the condition (C2) in Lemma 3.42 and the sequence of numbers $\left\{\prod_{i=1}^{n} a_{i}\right\}$ has a positive lower bound, we conclude that the inequality above leads to a contradiction that the right side tends to $\infty$ while $x(t)$ is bounded. Therefore, case $(B)$ holds. $x^{\prime}(t)<0$ implies that $x(t)$ is strictly monotonically decreasing. From the facts that the series $\sum_{n=1}^{\infty}\left|a_{i}-1\right|$ converges and $x(t)$ is bounded, it follows that $\sum_{i=1}^{\infty}\left[x\left(t_{i}^{+}\right)-x\left(t_{i}\right)\right]$ converges and there exists

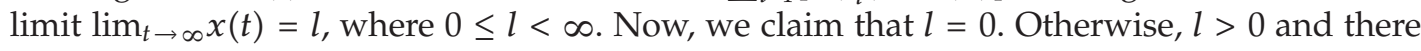
exists $T_{1} \geq T_{0}$ such that $x(t)>l / 2$ for $t \geq T_{1}$. From (3.91) and the last inequality, we can deduce

$$
\begin{gathered}
x^{\prime \prime \prime}(t)=-p(t) x(t)<-\frac{l}{2} p(t), \\
\int_{t_{s}}^{t_{s+n}} t^{2} x^{\prime \prime \prime}(t) d t \leq-\frac{l}{2} \int_{t_{s}}^{t_{s+n}} t^{2} p(t) d t .
\end{gathered}
$$


Integrating by parts of the above inequality and considering $b_{n} \leq 1, c_{n} \leq 1(n \in \mathbb{N})$, and $x^{\prime}(t)<0, x^{\prime \prime}(t)>0$, we have the following inequality:

$$
-\frac{l}{2} \int_{t_{s}}^{t_{s+n}} t^{2} p(t) d t \geq 2 x\left(t_{s+n}\right)+2 \sum_{i=1}^{n-1}\left(1-a_{s+i}\right) x\left(t_{s+i}\right)-\left[t_{s}^{2} x^{\prime \prime}\left(t_{s}^{+}\right)-2 t_{s} x^{\prime}\left(t_{s}^{+}\right)+2 x\left(t_{s}^{+}\right)\right] .
$$

Since $\int^{\infty} t^{2} p(t) d t=\infty$ and the series $\sum_{i=1}^{\infty}\left(1-a_{i}\right) x\left(t_{i}\right)$ converges, the above inequality contradicts the fact that $x(t)$ is bounded, hence $l=0$, and the proof is complete.

The proof of the following theorem is similar.

Theorem 3.44 (see [22]). Assume that conditions of Lemma 3.42 hold and for any $n \in \mathbb{N}, a_{n}>$ $0,1 \geq b_{n}>0,1 \geq c_{n}>0$, and $a_{n} / c_{n} \geq t_{n+1} / t_{n}$. Moreover, assume that the sequence of numbers $\left\{\prod_{i=1}^{n} a_{i}\right\}$ is bounded above, $\sum_{n=1}^{\infty}\left|a_{n}-1\right|$ converges, and $\int^{\infty} t p(t) d t=\infty$ holds. Then every solution of (3.91) either oscillates or tends asymptotically to zero with fixed sign.

Some results similar to the above theorems have been obtained for fourth-order linear impulsive differential equations; see [24].

\section{Nonlinear Equations}

In this section we present several oscillation theorems known for super-liner, half-linear, super-half-linear, and fully nonlinear impulsive differential equations of second and higherorders. We begin with Sturmian and Leighton type comparison theorems for half-linear equations.

\subsection{Sturmian Theory for Half-Linear Equations}

Consider the second-order half linear impulsive differential equations of the form:

$$
\begin{gathered}
\left(k(t) \varphi\left(x^{\prime}\right)\right)^{\prime}+p(t) \varphi(x)=0, \quad t \neq \theta_{i}, \\
\left.\Delta x\right|_{t=\theta_{i}}=0,\left.\quad \Delta\left(k(t) \varphi\left(x^{\prime}\right)\right)\right|_{t=\theta_{i}}+p_{i} \varphi(x)=0, \\
\left(m(t) \varphi\left(y^{\prime}\right)\right)^{\prime}+q(t) \varphi(y)=0, \quad t \neq \theta_{i}, \\
\left.\Delta y\right|_{t=\theta_{i}}=0,\left.\quad \Delta\left(m(t) \varphi\left(y^{\prime}\right)\right)\right|_{t=\theta_{i}}+q_{i} \varphi(y)=0,
\end{gathered}
$$

where $\varphi(s)=|s|^{\alpha-1} s, \alpha>0, \theta_{i}<\theta_{i+1},\left\{p_{i}\right\}$ and $\left\{q_{i}\right\}$ are real sequences, and $k, m, p, q \in \operatorname{PLC}(J)$ with $k(t)>0$ and $m(t)>0$.

The lemma below can be found in [26].

Lemma 4.1. Let $A, B \in \mathbb{R}$ and $\gamma>0$ be a constant; then

$$
A \varphi_{\gamma}(A)+\gamma B \varphi(B)-(\gamma+1) A \varphi(B) \geq 0,
$$

where equality holds if and only if $A=B$. 
The results of this section are from [16].

Theorem 4.2 (Sturm-Picone type comparison). Let $x(t)$ be a solution of (4.1) having two consecutive zeros $a$ and $b$ in $J$. Suppose that $p(t) \leq q(t)$ and $m(t) \leq k(t)$ are satisfied for all $t \in[a, b]$, and that $p_{i} \leq q_{i}$ for all $i \in \mathbb{N}$ for which $\theta_{i} \in[a, b]$. If either $p(t) \not \equiv q(t)$ or $k(t) \neq \equiv(t)$ or $p_{i} \not \equiv q_{i}$, then any solution $y(t)$ of (4.2) must have at least one zero in $(a, b)$.

Proof. Assume that $y(t)$ never vanishes on $(a, b)$. Define

$$
u(t):=\frac{x}{\varphi(y)}\left[k(t) \varphi(y) \varphi\left(x^{\prime}\right)-m(t) \varphi(x) \varphi\left(y^{\prime}\right)\right]
$$

where the dependence on $t$ of the solutions $x$ and $y$ is suppressed. It is not difficult to see that

$$
\begin{aligned}
u^{\prime}(t)= & \{k(t)-m(t)\}\left|x^{\prime}\right|^{\alpha+1}+\{q(t)-p(t)\}|x|^{\alpha+1}+m(t) \\
& \times\left[x^{\prime} \varphi\left(x^{\prime}\right)+\alpha\left(\frac{x y^{\prime}}{y}\right) \varphi\left(\frac{x y^{\prime}}{y}\right)-(\alpha+1) x^{\prime} \varphi\left(\frac{x y^{\prime}}{y}\right)\right], \quad t \neq \theta_{i}, \\
\left.\Delta u\right|_{t=\theta_{i}}= & \left(q_{i}-p_{i}\right)\left|x\left(\theta_{i}\right)\right|^{\alpha+1} .
\end{aligned}
$$

Clearly, the last term of (4.5) is integrable over $(a, b)$ if $y(a) \neq 0$ and $y(b) \neq 0$. Moreover, $u\left(a^{+}\right)=u\left(b^{-}\right)=0$ in this case. Suppose that $y\left(a^{+}\right)=0$. The case $y\left(b^{-}\right)=0$ is similar. Since $y^{\prime}\left(a^{+}\right) \neq 0$ and

$$
\lim _{t \rightarrow a^{+}} \frac{x(t)}{y(t)}=\lim _{t \rightarrow a^{+}} \frac{x^{\prime}(t)}{y^{\prime}(t)}<\infty
$$

we get

$$
\lim _{t \rightarrow a^{+}} \varphi\left(\frac{x(t)}{y(t)}\right)<\infty
$$

and so

$$
\begin{aligned}
\lim _{t \rightarrow a^{+}} & {\left[x^{\prime} \varphi\left(x^{\prime}\right)+\alpha\left(\frac{x y^{\prime}}{y}\right) \varphi\left(\frac{x y^{\prime}}{y}\right)-(\alpha+1) x^{\prime} \varphi\left(\frac{x y^{\prime}}{y}\right)\right] } \\
& =\lim _{t \rightarrow a^{+}}\left[x^{\prime} \varphi\left(x^{\prime}\right)+\alpha\left(\frac{x}{y}\right) \varphi\left(\frac{x}{y}\right) y^{\prime} \varphi\left(y^{\prime}\right)-(\alpha+1) x^{\prime} \varphi\left(y^{\prime}\right) \varphi\left(\frac{x}{y}\right)\right]<\infty .
\end{aligned}
$$

Moreover,

$$
\lim _{t \rightarrow a^{+}} u(t)=\lim _{t \rightarrow a^{+}} x\left[k(t) \varphi\left(x^{\prime}\right)-m(t) \varphi\left(\frac{x}{y}\right) \varphi\left(y^{\prime}\right)\right]=0 .
$$


Integrating (4.5) from $a$ to $b$ and using (4.6), we see that

$$
\begin{aligned}
0= & \int_{a}^{b}\left[(k(t)-m(t))\left|x^{\prime}(t)\right|^{\alpha+1}+(q(t)-p(t))|x(t)|^{\alpha+1}\right] d t \\
& +\int_{a}^{b} m(t)\left[x^{\prime} \varphi\left(x^{\prime}\right)+\alpha\left(\frac{x y^{\prime}}{y}\right) \varphi\left(\frac{x y^{\prime}}{y}\right)-(\alpha+1) x^{\prime} \varphi\left(\frac{x y^{\prime}}{y}\right)\right] d t+\sum_{a<\theta_{i}<b}\left(q_{i}-p_{i}\right)\left|x\left(\theta_{i}\right)\right|^{\alpha+1},
\end{aligned}
$$

where we have used Lemma 4.1 with $A=x^{\prime}, B=x y^{\prime} / y, \gamma=\alpha$, and $\varphi_{\alpha}(s)=\varphi(s)$. It is clear that (4.11) is not possible under our assumptions, and hence $y(t)$ must have a zero in $(a, b)$.

Corollary 4.3 (Separation Theorem). The zeros of two linearly independent solutions $x(t)$ and $y(t)$ of (4.1) separate each other.

Corollary 4.4 (Comparison Theorem). Suppose that $p(t) \leq q(t)$ and $m(t) \leq k(t)$ are satisfied for all $t \in\left[t_{*}, \infty\right)$ for some $t_{*} \geq t_{0}$, and that $p_{i} \leq q_{i}$ for all $i \in \mathbb{N}$ for which $\theta_{i} \geq t_{*}$. If either $p(t) \neq \equiv q(t)$ or $k(t) \not \equiv m(t)$ or $p_{i} \not \equiv q_{i}$, then every solution $y(t)$ of (4.2) is oscillatory whenever a solution $x(t)$ of (4.1) is oscillatory.

Corollary 4.5 (Dichotomy Theorem). The solutions of (4.1) are either all oscillatory or all nonoscillatory.

Theorem 4.6 (Leighton-type Comparison). Let $x(t)$ be a solution of (4.1) having two consecutive zeros $a$ and $b$ in $J$. Suppose that

$$
V_{\alpha}[x]:=\int_{a}^{b}\left[\{q(t)-p(t)\}|x(t)|^{\alpha+1}+\{k(t)-m(t)\}\left|x^{\prime}(t)\right|^{\alpha+1}\right] d t+\sum_{a<\theta_{i}<b}\left(q_{i}-p_{i}\right)\left|x\left(\theta_{i}\right)\right|^{\alpha+1}>0 .
$$

Then any nontrivial solution $y(t)$ of (4.2) must have at least one zero in $(a, b)$.

Proof. Assume that $y(t)$ has no zero in $(a, b)$. Define the function $u(t)$ as in (4.4).

Clearly, (4.5) and (4.6) hold. It follows that

$$
\begin{aligned}
0= & u\left(b^{-}\right)-u\left(a^{+}\right) \\
= & \int_{a}^{b}\left[(k(t)-m(t))\left|x^{\prime}\right|^{\alpha+1}+(q(t)-p(t))|x|^{\alpha+1}\right] d t \\
& +\int_{a}^{b} m(t)\left[x^{\prime} \varphi\left(x^{\prime}\right)+\alpha\left(\frac{x y^{\prime}}{y}\right) \varphi\left(\frac{x y^{\prime}}{y}\right)-(\alpha+1) x^{\prime} \varphi\left(\frac{x y^{\prime}}{y}\right)\right] d t+\sum_{a<\theta_{i}<b}\left|x\left(\theta_{i}\right)\right|^{\alpha+1}\left(q_{i}-p_{i}\right) \\
= & V_{\alpha}[x]+\int_{a}^{b} m(t)\left[x^{\prime} \varphi\left(x^{\prime}\right)+\alpha\left(\frac{x y^{\prime}}{y}\right) \varphi\left(\frac{x y^{\prime}}{y}\right)-(\alpha+1) x^{\prime} \varphi\left(\frac{x y^{\prime}}{y}\right)\right] d t>0,
\end{aligned}
$$

which is a contradiction. Therefore, $y(t)$ must have a zero on $(a, b)$. 
If $V_{\alpha}[x] \geq 0$, then we may conclude that either $y(t)$ has a zero in $(a, b)$ or $y(t)$ is a constant multiple of $x(t)$.

As a consequence of Theorems 4.2 and 4.6, we have the following oscillation result.

Corollary 4.7. Suppose for a given $T \geq t_{*}$ there exists an interval $(a, b) \subset[T, \infty)$ for which either conditions of Theorem 4.2 or Theorem 4.6 are satisfied, then every solution $y(t)$ of (4.2) is oscillatory.

\subsection{Oscillation of Second-Order Superlinear and Super-Half-Linear Equations}

Let us consider the forced superlinear second-order differential equation of the following form:

$$
\begin{gathered}
\left(r(t) x^{\prime}\right)^{\prime}+p(t)|x|^{\alpha-1} x=q(t), \quad t \neq \tau_{k}, \\
x\left(\tau_{k}^{+}\right)=a_{k} x\left(\tau_{k}\right), \quad x^{\prime}\left(\tau_{k}^{+}\right)=b_{k} x^{\prime}\left(\tau_{k}\right), \quad k \in \mathbb{N}, \\
x\left(t_{0}^{+}\right)=x_{0}, \quad x^{\prime}\left(t_{0}^{+}\right)=x_{0}^{\prime},
\end{gathered}
$$

where $\left\{\tau_{k}\right\}$ denotes the impulse moments sequence with $0 \leq t_{0}=\tau_{0}<\tau_{1}<\cdots<\tau_{k}$ $<\cdots, \lim _{k \rightarrow \infty} \tau_{k}=\infty$.

Assume that the following conditions hold.

$(A 1) \alpha>1$ is a constant, $r: J \rightarrow(0, \infty)$ is a continuous function, $p, q \in C(J)$.

$(A 2) b_{k} \geq a_{k}>0$ are constants, $k \in \mathbb{N}$.

(A3) $\left[c_{1}, d_{1}\right]$ and $\left[c_{2}, d_{2}\right]$ are two intervals such that $c_{j}, d_{j} \notin\left\{\tau_{k}\right\}(j=1,2)$ with $c_{1}<d_{1} \leq$ $c_{2}<d_{2}, p(t) \geq 0$ for $t \in\left[c_{1}, d_{1}\right] \cup\left[c_{2}, d_{2}\right]$, and $q(t) \leq 0$ for $t \in\left[c_{1}, d_{1}\right], q(t) \geq 0$ for $t \in\left[c_{2}, d_{2}\right]$.

Interval oscillation criteria for (4.14) are given in [27]. Denote $k(s)=\max \left\{i: t_{0}<\tau_{i}<\right.$ $s\}$, and for $j=1,2$, let $\eta_{j}=\max \left\{r(t): t \in\left[c_{j}, d_{j}\right]\right\}$,

$$
\begin{gathered}
\Omega_{w}\left(c_{j}, d_{j}\right)=\left\{w \in C^{1}\left[c_{j}, d_{j}\right], w(t) \not \equiv 0, w\left(c_{j}\right)=w\left(d_{j}\right)=0\right\}, \\
\Omega_{G}\left(c_{j}, d_{j}\right)=\left\{G \in C^{1}\left[c_{j}, d_{j}\right], G(t) \geq 0, \not \equiv 0, G\left(c_{j}\right)=G\left(d_{j}\right)=0, G(t)=2 g(t) \sqrt{G(t)}\right\} .
\end{gathered}
$$

Theorem 4.8 (see [27]). Assume that conditions (A1)-(A3) hold, and that there exists $w(t) \in$ $\Omega_{w}\left(c_{j}, d_{j}\right)$ such that

$$
\int_{c_{j}}^{d_{j}}\left\{[p(t)]^{1 / \alpha}|q(t)|^{1-1 / \alpha} w^{2}(t)-r(t) w^{\prime 2}(t)\right\} d t \geq Q\left(w, c_{j}, d_{j}\right),
$$

where $Q\left(w, c_{j}, d_{j}\right)=0$ for $k\left(c_{j}\right)=k\left(d_{j}\right)$, and

$$
Q\left(w, c_{j}, d_{j}\right)=\eta_{j}\left\{w^{2}\left(\tau_{k\left(c_{j}\right)+1}\right) \frac{b_{k\left(c_{j}\right)+1}-a_{k\left(c_{j}\right)+1}}{a_{k\left(c_{j}\right)+1}\left(\tau_{k\left(c_{j}\right)+1}-c_{j}\right)}+\sum_{i=k\left(c_{j}\right)+2}^{k\left(d_{j}\right)} w^{2}\left(\tau_{i}\right) \frac{b_{i}-a_{i}}{a_{i}\left(\tau_{i}-\tau_{i-1}\right)}\right\}
$$

for $k\left(c_{j}\right)<k\left(d_{j}\right), j=1,2$. Then every solution of (4.14) has at least one zero in $\left[c_{1}, d_{1}\right] \cup\left[c_{2}, d_{2}\right]$. 
Proof. Let $x(t)$ be a solution of (4.14). Suppose that $x(t)$ does not have any zero in $\left[c_{1}, d_{1}\right] \cup$ $\left[c_{2}, d_{2}\right]$. Without loss of generality, we may assume that $x(t)>0$ for $t \in\left[c_{1}, d_{1}\right]$. Define

$$
u(t)=-\frac{r(t) x^{\prime}(t)}{x(t)}, \quad t \in\left[c_{1}, d_{1}\right]
$$

Then, by Hölder's inequality, for $t \in\left[c_{1}, d_{1}\right]$ and $t \neq \tau_{k}$, we have

$$
\begin{aligned}
u^{\prime}(t) & =p(t)[x(t)]^{\alpha-1}-\frac{q(t)}{x(t)}+\frac{1}{r(t)} u^{2}(t) \\
& \geq[p(t)]^{1 / \alpha}|q(t)|^{1-1 / \alpha}+\frac{1}{r(t)} u^{2}(t), \quad t \in\left(c_{1}, d_{1}\right), t \neq \tau_{k}
\end{aligned}
$$

For $t=\tau_{k}, k \in \mathbb{N}$, we obtain

$$
u\left(\tau_{k}^{+}\right)=\frac{b_{k}}{a_{k}} u\left(\tau_{k}\right)
$$

If $k\left(c_{1}\right)<k\left(d_{1}\right)$, then all impulsive moments are in $\left[c_{1}, d_{1}\right]: \tau_{k\left(c_{1}\right)+1}, \tau_{k\left(c_{1}\right)+2}, \ldots, \tau_{k\left(d_{1}\right)}$. Multiplying both sides of (4.19) by $w^{2}(t)$ and integrating on $\left[c_{1}, d_{1}\right]$ and using the hypotheses, we get

$$
\sum_{i=k\left(c_{1}\right)+1}^{k\left(d_{1}\right)} \frac{a_{i}-b_{i}}{a_{i}} w^{2}\left(\tau_{i}\right) u\left(\tau_{i}\right)>\int_{c_{1}}^{d_{1}}\left\{[p(t)]^{1 / \alpha}|q(t)|^{1-1 / \alpha} w^{2}(t)-r(t) w^{\prime 2}(t)\right\} d t .
$$

On the other hand, for $t \in\left(c_{1}, \tau_{k\left(c_{1}\right)+1}\right]$, it follows that

$$
\left(r(t) x^{\prime}(t)\right)^{\prime}=q(t)-p(t)|x(t)|^{\alpha-1} x(t) \leq 0,
$$

which implies that $r(t) x^{\prime}(t)$ is nonincreasing on $\left(c_{1}, \tau_{k\left(c_{1}\right)+1}\right]$. So, for any $t \in\left(c_{1}, \tau_{k\left(c_{1}\right)+1}\right]$, one has

$$
x(t)-x\left(c_{1}\right) \geq \frac{r(t) x^{\prime}(t)}{r(\xi)}\left(t-c_{1}\right), \quad \xi \in\left(c_{1}, t\right) .
$$

It follows from the above inequality that

$$
u\left(\tau_{k\left(c_{1}\right)+1}\right) \geq-\frac{r(\xi)}{\tau_{k\left(c_{1}\right)+1}-c_{1}} \geq-\frac{\eta_{1}}{\tau_{k\left(c_{1}\right)+1}-c_{1}} .
$$

Making a similar analysis on $\left(\tau_{i-1}, \tau_{i}\right], i=k\left(c_{1}\right)+2, \ldots, k\left(d_{1}\right)$, we obtain

$$
u\left(\tau_{i}\right)=-\frac{r\left(\tau_{i}\right) x^{\prime}\left(\tau_{i}\right)}{x\left(\tau_{i}\right)} \geq-\frac{\eta_{1}}{\tau_{i}-\tau_{i-1}}, \quad i=k\left(c_{1}\right)+2, \ldots, k\left(d_{1}\right) .
$$


From (4.21)-(4.25) and (A2), we get

$$
\int_{c_{1}}^{d_{1}}\left[[p(t)]^{1 / \alpha}|q(t)|^{1-1 / \alpha} w^{2}(t)-r(t) w^{\prime 2}(t)\right] d t<Q\left(w, c_{1}, d_{1}\right)
$$

which contradicts (4.16). If $k\left(c_{1}\right)=k\left(d_{1}\right)$, then $Q\left(w, c_{1}, d_{1}\right)=0$, and there are no impulse moments in $\left[c_{1}, d_{1}\right]$. Similarly to the proof of $(4.21)$, we get

$$
0>\int_{c_{1}}^{d_{1}}\left\{[p(t)]^{1 / \alpha}|q(t)|^{1-1 / \alpha} w^{2}(t)-r(t) w^{\prime 2}(t)\right\} d t
$$

which again contradicts (4.16).

In the case $x(t)<0$, one can repeat the above procedure on the subinterval $\left[c_{2}, d_{2}\right]$ in place of $\left[c_{1}, d_{1}\right]$. This completes the proof.

Corollary 4.9. Assume that conditions (A1) and (A2) hold. If for any $T>0$ there exist $c_{j}, d_{j}$ satisfying (A3) with $T \leq c_{1}<d_{1} \leq c_{2}<d_{2}$, and $w(t) \in \Omega_{w}\left(c_{j}, d_{j}\right)$ satisfying (4.16), $j=1,2$, then (4.14) is oscillatory.

The proof of following theorem is similar to that of Theorem 4.8.

Theorem 4.10 (see [27]). Assume that conditions (A1)-(A3) hold, and there exists a $G \in \Omega_{G}\left(c_{j}, d_{j}\right)$ such that

$$
\int_{c_{j}}^{d_{j}}\left\{[p(t)]^{1 / \alpha}|q(t)|^{1-1 / \alpha} G(t)-r(t) h^{2}(t)\right\} d t>R\left(G, c_{j}, d_{j}\right),
$$

where $R\left(G, c_{j}, d_{j}\right)=0$ for $k\left(c_{j}\right)=k\left(d_{j}\right)$, and

$$
R\left(G, c_{j}, d_{j}\right)=\eta_{j}\left[G\left(\tau_{k\left(c_{j}\right)+1}\right) \frac{b_{k\left(c_{j}\right)+1}-a_{k\left(c_{j}\right)+1}}{a_{k\left(c_{j}\right)+1}\left(\tau_{k\left(c_{j}\right)+1}-c_{j}\right)}+\sum_{i=k\left(c_{j}\right)+2}^{k\left(d_{j}\right)} G\left(\tau_{i}\right) \frac{b_{i}-a_{i}}{a_{i}\left(\tau_{i}-\tau_{i-1}\right)}\right]
$$

for $k\left(c_{j}\right)<k\left(d_{j}\right), j=1,2$. Then every solution of (4.14) has at least one zero in $\left[c_{1}, d_{1}\right] \cup\left[c_{2}, d_{2}\right]$.

Corollary 4.11. Assume that conditions (A1) and (A2) hold. If for any $T>0$ there exist $c_{j}, d_{j}$ satisfying (A3) with $T \leq c_{1}<d_{1} \leq c_{2}<d_{2}$, and $G \in \Omega_{G}\left(c_{j}, d_{j}\right)$ satisfying (4.28), $j=1,2$, then (4.14) is oscillatory.

Example 4.12. Consider the following superlinear impulsive differential equation:

$$
\begin{gathered}
\left(\left(1+\sin ^{2} t\right) x^{\prime}\right)^{\prime}+(\beta \cos t)|x|^{\alpha-1} x=\sin t, \quad t \neq 2 k \pi \pm \frac{\pi}{4}, \\
x\left(\tau_{k}^{+}\right)=a_{k} x\left(\tau_{k}\right), \quad x^{\prime}\left(\tau_{k}^{+}\right)=b_{k} x^{\prime}\left(\tau_{k}\right), \quad \tau_{k}=2 k \pi \pm \frac{\pi}{4}, \quad k \in \mathbb{N} .
\end{gathered}
$$


It can be seen that if

$$
\beta^{1 / \alpha} \frac{\Gamma(3+1 / \alpha) \Gamma(4+1 / \alpha)}{\Gamma(7)} \geq \frac{3 \pi}{4}+\frac{2\left(b_{k}-a_{k}\right)}{\pi}, \quad k \in \mathbb{N}
$$

then, conditions of Corollary 4.9 are satisfied; here $\alpha>1, \Gamma$ is the gamma function, and $a_{k}$ and $b_{k}$ satisfy $(A 2)$. So, every solution of (4.30) is oscillatory.

In [28-30], the authors have used an energy function approach to obtain conditions for the existence of oscillatory or nonoscillatory solutions of the half-linear impulsive differential equations of the following form:

$$
\begin{gathered}
\left(\phi_{\beta}\left(x^{\prime}\right)\right)^{\prime}+\phi_{\beta}(x)=0, \quad t \neq t_{n}, \\
x\left(t_{n}+0\right)=x\left(t_{n}\right), \quad x^{\prime}\left(t_{n}+0\right)=b_{n} x^{\prime}\left(t_{n}\right),
\end{gathered}
$$

where $b_{n} \geq 1$ for $n \in \mathbb{N}$.

Define the energy functional

$$
V(x, y)=y \phi_{\beta}(y)-\int_{0}^{y} \phi_{\beta}(s) d s+\int_{0}^{x} \phi_{\beta}(s) d s=: \Phi_{\beta}(y)+F_{\beta}(x)
$$

where in explicit form $\Phi_{\beta}(y)=(\beta /(\beta+1))|y|^{\beta+1}$ and $F_{\beta}(x)=(1 /(\beta+1))|x|^{\beta+1}$. The functions $\Phi_{\beta}$ and $F_{\beta}$ are both even and positive definite. equation

The function $V(t)=V\left(x(t), x^{\prime}(t)\right)$ is constant along the solutions of the nonimpulsive

$$
\left(\phi_{\beta}\left(x^{\prime}\right)\right)^{\prime}+\phi_{\beta}(x)=0 .
$$

The change in the energy along the solutions of (4.32) is given by

$$
\begin{aligned}
V\left(t_{n+1}\right)-V\left(t_{n}\right) & =V\left(t_{n}+0\right)-V\left(t_{n}\right) \\
& =\Phi_{\beta}\left(x^{\prime}\left(t_{n}+0\right)\right)+F_{\beta}\left(x\left(t_{n}+0\right)\right)-\Phi_{\beta}\left(x^{\prime}\left(t_{n}\right)\right)-F_{\beta}\left(x\left(t_{n}\right)\right) \\
& =b_{n}^{\beta+1} \Phi_{\beta}\left(x^{\prime}\left(t_{n}\right)\right)-\Phi_{\beta}\left(x^{\prime}\left(t_{n}\right)\right)=\Phi_{\beta}\left(x^{\prime}\left(t_{n}\right)\right)\left(b_{n}^{\beta+1}-1\right) .
\end{aligned}
$$

We see that these impulsive perturbations increase the energy. If the energy increases slowly, then we expect the solutions to oscillate. On the other hand, if the energy increases too fast, the solutions become nonoscillatory. Let $x(t)$ be a solution of $(4.32), r_{n}=V\left(t_{n-1}+0\right)=V\left(t_{n}-0\right)$, and $F_{\beta}\left(x\left(t_{n}\right)\right)=\sigma_{n} r_{n}$. Calculating $F_{\beta}\left(x\left(t_{n}\right)\right)$ in terms of $r_{n+1}=V\left(t_{n}+0\right)$, we obtain

$$
F_{\beta}\left(x\left(t_{n}\right)\right)=\frac{\sigma_{n}}{b_{n}^{\beta+1}\left(1-\sigma_{n}\right)+\sigma_{n}} r_{n+1} .
$$


To simplify the notation, we introduce the function

$$
\Theta(u, b):=\frac{u}{b^{\beta+1}(1-u)+u} .
$$

The function $\Theta$ gives the jump in the quantity $F_{\beta}(x(t)) / V(t)$. Note that $\Theta(0, b)=0,0<$ $\Theta(u, b)<u$ for $0<u<1$ and $b \geq 1$; $\Theta$ is monotone increasing with respect to $u$ and decreasing with respect to $b$.

Theorem 4.13 (see [29]). Assume that there exist a constant $N>0$ and a sequence $\left\{\gamma_{n}\right\}$ with $\Theta\left(\gamma_{n}, b_{n}\right) \leq \gamma_{n+1}<1$ such that

$$
t_{n+1}-t_{n} \leq \int_{\Theta\left(\gamma_{n}, \beta_{n}\right)}^{\gamma_{n+1}} \frac{d v}{\phi_{\beta}\left(F_{\beta}^{-1}(v)\right) \Phi_{\beta}^{-1}(1-v)}
$$

holds for every $n>N$. Then every solution of (4.32) is nonoscillatory.

Theorem 4.14 (see [29]). Assume that there exist a constant $N>0$ and a sequence $\left\{\lambda_{n}\right\}$ with $\lambda_{n}>0$ and $\sum_{n=1}^{\infty} \lambda_{n}=\infty$ such that for every $\gamma \in[0,1]$

$$
t_{n+1}-t_{n} \geq \int_{\Theta\left(\gamma, b_{n}\right)}^{\gamma+\lambda_{n}} \frac{d v}{\phi_{\beta}\left(F_{\beta}^{-1}(v)\right) \Phi_{\beta}^{-1}(1-v)}
$$

holds for every $n>N$. Then every solution of (4.32) is oscillatory.

Proof. Let $x(t)$ be a nontrivial solution of (4.32). It suffices to show that $x(t) x^{\prime}(t)>0$ cannot hold on any interval $[T, \infty)$. Assume that to the contrary, $x(t)>0, x^{\prime}(t)>0$ for $t \in\left[t_{N}, \infty\right)$. Let $\sigma_{n}$ be defined by $F_{\beta}\left(x\left(t_{n}\right)\right)=\sigma_{n} r_{n}$, where $r_{n}=V\left(t_{n}-0\right)$. It follows from (4.39) that $\sigma_{n+1} \geq \sigma_{n}+\lambda_{n}$. Hence,

$$
\sigma_{n+1} \geq \sigma_{N}+\sum_{i=N}^{\mathrm{n}} \lambda_{n}
$$

Since $\sigma_{n}=F_{\beta}\left(x\left(t_{n}\right)\right) / r_{n} \leq 1$, and the right side of the above inequality tends to infinity as $n \rightarrow \infty$, we have a contradiction.

Now, assume that $b_{n} \geq b>1$ for every $n>N$. It can be shown that the integral

$$
\int_{\Theta(r, b)}^{\gamma} \frac{d v}{\phi_{\beta}\left(F_{\beta}^{-1}(v)\right) \Phi_{\beta}^{-1}(1-v)}
$$

takes its maximum in $[0,1]$ at

$$
\bar{\gamma}=\frac{b^{\beta}(b-1)}{b^{\beta+1}-1}
$$


In the special case $b_{n}=b, t_{n+1}-t_{n}=d(n \in \mathbb{N})$, we have the following necessary and sufficient condition.

Theorem 4.15 (see [29]). Assume that $b_{n}=b, t_{n+1}-t_{n}=d(n \in \mathbb{N})$. Then every solution of (4.32) is nonoscillatory if and only if

$$
d \leq \int_{\Theta(\bar{\gamma}, b)}^{\bar{\gamma}} \frac{d v}{\phi_{\beta}\left(F_{\beta}^{-1}(v)\right) \Phi_{\beta}^{-1}(1-v)} .
$$

Remark 4.16. Equation (4.32) with $0 \leq b_{n} \leq 1$, was studied in [30]. form:

Finally, we consider the second-order impulsive differential equation of the following

$$
\begin{gathered}
\left(r(t) \varphi_{\alpha}\left(x^{\prime}\right)\right)^{\prime}+p(t) \varphi_{\alpha}\left(x^{\prime}\right)+q(t) \varphi_{\beta}(x)=e(t), \quad t \neq \theta_{i}, \\
\Delta\left(r(t) \varphi_{\alpha}\left(x^{\prime}\right)\right)+q_{i} \varphi_{\beta}(x)=e_{i}, \quad t=\theta_{i},
\end{gathered}
$$

where $\varphi_{\gamma}(s)=|s|^{\gamma-1} s, \beta \geq \alpha>0$ are real constants, $\left\{\theta_{i}\right\}$ is a strictly increasing unbounded sequence of real numbers, $\left\{q_{i}\right\}$ and $\left\{e_{i}\right\}$ are real sequences, $r, p, q, e \in \operatorname{PLC}(J)$, and $r(t)>0$.

All results given in the remainder of this section are from [31].

Theorem 4.17. Suppose that for any given $t_{*} \geq t_{0}$, there exist intervals $I_{1}=\left[s_{1}, t_{1}\right], I_{2}=\left[s_{2}, t_{2}\right] \subset$ $\left[t_{*}, \infty\right)$, such that

(a) $q(t) \geq 0$ for all $t \in\left\{I_{1} \cup I_{2}\right\} \backslash\left\{\theta_{i}\right\}$ and $q_{i} \geq 0$ for all $i \in \mathbb{N}$ for which $\theta_{i} \in I_{1} \cup I_{2}$;

(b) $e(t) \leq 0, t \in I_{1} \backslash\left\{\theta_{i}\right\}, e(t) \geq 0, t \in I_{2} \backslash\left\{\theta_{i}\right\} ; e_{i} \leq 0, \theta_{i} \in I_{1}, e_{i} \geq 0, \theta_{i} \in I_{2}$ for all $i \in \mathbb{N}$.

If there exists $H \in \Phi\left(s_{k}, t_{k}\right)$ such that

$$
\begin{gathered}
\int_{s_{k}}^{t_{k}}\left\{\tilde{q}(t)|H(t)|^{\alpha+1}-(\alpha+1)^{-(\alpha+1)} r(t)\left|(\alpha+1) H^{\prime}(t)-\frac{p(t)}{r(t)} H(t)\right|^{\alpha+1}\right\} d t \\
+\sum_{s_{k} \leq \theta_{i}<t_{k}} \tilde{q}_{i}\left|H\left(\theta_{i}\right)\right|^{\alpha+1}>0, \quad(k=1,2),
\end{gathered}
$$

where

$$
\begin{gathered}
\tilde{q}(t)=\beta \alpha^{-\alpha / \beta}(\beta-\alpha)^{\alpha / \beta-1}[q(t)]^{\alpha / \beta}|e(t)|^{1-\alpha / \beta}, \\
\tilde{q}_{i}=\beta \alpha^{-\alpha / \beta}(\beta-\alpha)^{\alpha / \beta-1}\left[q_{i}\right]^{\alpha / \beta}\left|e_{i}\right|^{1-\alpha / \beta},
\end{gathered}
$$

then (4.44) is oscillatory. 
Proof. Suppose that there exists a nonoscillatory solution $x(t)$ of $(4.44)$ so that $x(t) \neq 0$ for all $t \geq t_{*}$ for some $t_{*}$. Let

$$
\mathcal{v}(t):=\frac{r(t) \varphi_{\alpha}\left(x^{\prime}(t)\right)}{\varphi_{\alpha}(x(t))}, \quad t \geq t_{*}
$$

It follows that for $t \geq t_{*}$,

$$
\begin{gathered}
v^{\prime}=-\frac{p}{r} v-\alpha \frac{|v|^{1+1 / \alpha}}{r^{1 / \alpha}}-\left(q|x|^{\beta-\alpha}-\frac{e}{\varphi_{\alpha}(x)}\right), \quad t \neq \theta_{i} ; \\
\Delta v=-\left(q_{i}|x|^{\beta-\alpha}-\frac{e_{i}}{\varphi_{\alpha}(x)}\right), \quad t=\theta_{i},
\end{gathered}
$$

where $t$ dependence is suppressed for clarity.

Define a function $E(z):(0, \infty) \rightarrow(0, \infty)$ by

$$
E(z):=A_{1} z^{\beta-\alpha}+A_{2} z^{-\alpha}, \quad A_{1} \geq 0, A_{2} \geq 0
$$

It is not difficult to see that if $\beta>\alpha$, then

$$
\min _{z \in(0, \infty)} E(z)=\beta \alpha^{-\alpha / \beta}(\beta-\alpha)^{(\alpha-\beta) / \beta} A_{1}^{\alpha / \beta} A_{2}^{(\beta-\alpha) / \beta}
$$

Clearly, if $\beta=\alpha$, then we have $E(z) \geq A_{1}$. Thus, with our convention that $0^{0}=1$, (4.51) holds for $\beta \geq \alpha$.

Suppose that $x(t)>0$ for all $t \geq t_{*}$. Choose $s_{1} \geq t_{*}$ and consider the interval $I_{1}=\left[s_{1}, t_{1}\right]$. From (b), we see that $e(t) \leq 0$ on $I_{1} \backslash\left\{\theta_{i}\right\}$ and $e_{i} \leq 0$ for all $i \in \mathbb{N}$ for which $\theta_{i} \in I_{1}$. Applying (4.51) to the terms in the parenthesis in (4.49) we obtain

$$
\begin{gathered}
\tilde{q} \leq-\mathcal{v}^{\prime}-\frac{p}{r} v-\alpha \frac{|v|^{1+1 / \alpha}}{r^{1 / \alpha}}, \quad t \in I_{1} \backslash\left\{\theta_{i}\right\} \\
\Delta v\left(\theta_{i}\right)+\tilde{q}_{i} \leq 0, \quad \theta_{i} \in I_{1}
\end{gathered}
$$

where $\tilde{q}$ and $\tilde{q}_{i}$ are defined by (4.46) and (4.47), respectively.

Let $H \in \boldsymbol{\Phi}\left(s_{1}, t_{1}\right)$. Multiplying (4.52) by $|H|^{\alpha+1}$ and integrating over $I_{1}$ give

$$
\int_{s_{1}}^{t_{1}} \tilde{q}|H|^{\alpha+1} \mathrm{~d} t \leq-\int_{s_{1}}^{t_{1}} \frac{p}{r}|H|^{\alpha+1} v \mathrm{~d} t-\alpha \int_{S_{1}}^{t_{1}} \frac{|v|^{1+1 / \alpha}}{r^{1 / \alpha}}|H|^{\alpha+1} \mathrm{~d} t-\int_{S_{1}}^{t_{1}}|H|^{\alpha+1} v^{\prime} \mathrm{d} t
$$


In view of (4.52) and the assumption $H\left(s_{1}\right)=H\left(t_{1}\right)=0$, employing the integration by parts formula in the last integral we have

$$
\begin{aligned}
& \int_{s_{1}}^{t_{1}} \tilde{\tilde{q}}|H|^{\alpha+1} \mathrm{~d} t+\sum_{s_{1} \leq \theta_{i}<t_{1}} \tilde{q}_{i}|H|^{\alpha+1} \\
& \quad \leq(\alpha+1) \int_{s_{1}}^{t_{1}} \varphi_{\alpha}(H) H^{\prime} v \mathrm{~d} t-\int_{s_{1}}^{t_{1}} \frac{p}{r}|H|^{\alpha+1} v \mathrm{~d} t-\alpha \int_{s_{1}}^{t_{1}} \frac{|v|^{1+1 / \alpha}}{r^{1 / \alpha}}|H|^{\alpha+1} \mathrm{~d} t \\
& \quad \leq \int_{s_{1}}^{t_{1}}\left[\left.\left.\left|(\alpha+1) \varphi_{\alpha}(H) H^{\prime}-\frac{p}{r}\right| H\right|^{\alpha+1}|| v\left|\mathrm{~d} t-\alpha \frac{|H|^{\alpha+1}}{r^{1 / \alpha}}\right| v\right|^{1+1 / \alpha}\right] \mathrm{d} t .
\end{aligned}
$$

We use Lemma 4.1 with

$$
\begin{gathered}
r=\frac{1}{\alpha}, \quad A=\alpha^{\alpha /(\alpha+1)} \frac{|H|^{\alpha}}{r^{1 /(\alpha+1)}}|v|, \\
B=\left.\left.\left(\frac{\alpha(\alpha+1)^{-(\alpha+1)} r}{|H|^{\alpha(\alpha+1)}}\right)^{\alpha /(\alpha+1)}\left|(\alpha+1) \varphi_{\alpha}(H) H^{\prime}-\frac{p}{r}\right| H\right|^{\alpha+1}\right|^{\alpha},
\end{gathered}
$$

to obtain

$$
\int_{s_{1}}^{t_{1}} \tilde{q}|H|^{\alpha+1} \mathrm{~d} t+\sum_{s_{1} \leq \theta_{i}<t_{1}} \tilde{q}_{i}|H|^{\alpha+1} \leq(\alpha+1)^{-(\alpha+1)} \int_{s_{1}}^{t_{1}} r\left|(\alpha+1) H^{\prime}-\frac{p}{r} H\right|^{\alpha+1} \mathrm{~d} t
$$

which obviously contradicts (4.45).

If $x(t)$ is eventually negative then we can consider $I_{2}$ and reach a similar contradiction. This completes the proof.

Example 4.18. Consider

$$
\begin{gathered}
\left(\left|x^{\prime}\right|^{-2 / 3} x^{\prime}\right)^{\prime}+(\cos 3 t)\left|x^{\prime}\right|^{-2 / 3} x^{\prime}+(\cos t)|x|^{-1 / 2} x=\sin 3 t, \quad t \neq \frac{i \pi}{12} \\
\Delta\left(\left|x^{\prime}\right|^{-2 / 3} x^{\prime}\right)+\gamma\left(\sec ^{3 / 2} t\right)|x|^{-1 / 2} x=\cos ^{3} t \sin 3 t, \quad t=\frac{i \pi}{12}
\end{gathered}
$$

where $\gamma$ is a positive real number.

Let $H(t)=\sin ^{2} 3 t, I_{1}=[(2 n+5 / 3) \pi,(2 n+2) \pi]$, and $I_{2}=[(2 n+2) \pi,(2 n+7 / 3) \pi]$. For any given $t_{*} \geq 0$ we may choose $n \in \mathbb{N}$ sufficiently large so that $2 n \pi \geq t_{*}$. Then conditions 
(a)-(b) are satisfied. It is also easy to see that, for $j=2 n+5 / 3$ and $j=2 n+2$,

$$
\begin{aligned}
\int_{j \pi}^{(j+1 / 3) \pi} & {\left[3.2^{-2 / 3} \cos ^{2 / 3} t\left|\sin ^{3} 3 t\right|-\frac{3^{4 / 3}}{16}\left|\sin ^{4 / 3} 6 t\right|(8-\sin 3 t)^{4 / 3}\right] d t } \\
& +3\left(\frac{\gamma}{2}\right)^{2 / 3} \sum_{j \pi \leq \theta_{i}<(j+1 / 3) \pi}\left|\sin ^{3} 3 \theta_{i}\right| \\
= & A_{j}+3\left(\frac{\gamma}{2}\right)^{2 / 3} \sum_{12 j \leq i<12 j+4}\left|\sin ^{3}\left(\frac{i \pi}{4}\right)\right| \\
= & A_{j}+3\left(\frac{\gamma}{2}\right)^{2 / 3}\left(1+\frac{1}{\sqrt{2}}\right),
\end{aligned}
$$

where $A_{2 n+5 / 3} \approx-2.17024$ and $A_{2 n+2} \approx-1.58213$. It follows from Theorem 4.17 that $(4.57)$ is oscillatory if

$$
r>2\left[\frac{1}{3}(\sqrt{2}-2) A_{2 n+5 / 3}\right]^{3 / 2} \approx 0.55172
$$

Note that if there is no impulse then the above integrals are negative, and therefore no conclusion can be drawn.

When $\beta=\alpha$, then (4.44) reduces to forced half-linear impulsive equation with damping

$$
\begin{gathered}
\left(r(t) \varphi_{\alpha}\left(x^{\prime}\right)\right)^{\prime}+p(t) \varphi_{\alpha}\left(x^{\prime}\right)+q(t) \varphi_{\alpha}(x)=e(t), \quad t \neq \theta_{i}, \\
\Delta\left(r(t) \varphi_{\alpha}\left(x^{\prime}\right)\right)+q_{i} \varphi_{\alpha}(x)=e_{i}, \quad t=\theta_{i} .
\end{gathered}
$$

Corollary 4.19. Suppose that for any given $t_{*} \geq t_{0}$, there exist intervals $I_{1}=\left[s_{1}, t_{1}\right], I_{2}=\left[s_{2}, t_{2}\right] \subset$ $\left[t_{*}, \infty\right)$ for which $(a)-(b)$ hold.

If there exists $H \in \Phi\left(s_{k}, t_{k}\right)$ such that

$$
\begin{gathered}
\int_{s_{k}}^{t_{k}}\left\{q(t)|H(t)|^{\alpha+1}-(\alpha+1)^{-(\alpha+1)} r(t)\left|(\alpha+1) H^{\prime}(t)-\frac{p(t)}{r(t)} H(t)\right|^{\alpha+1}\right\} \mathrm{d} t \\
+\sum_{s_{k} \leq \theta_{i}<t_{k}} q_{i}\left|H\left(\theta_{i}\right)\right|^{\alpha+1}>0 \quad(k=1,2),
\end{gathered}
$$

then (4.60) is oscillatory.

Taking $\alpha=1$ in (4.44), we have the forced superlinear impulsive equation with damping

$$
\begin{gathered}
\left(r(t) x^{\prime}\right)^{\prime}+p(t) x^{\prime}+q(t)|x|^{\beta-1} x=e(t), \quad t \neq \theta_{i}, \\
\Delta\left(r(t) x^{\prime}\right)+q_{i}|x|^{\beta-1} x=e_{i}, \quad t=\theta_{i} .
\end{gathered}
$$


Corollary 4.20. Let $\beta_{0}=\beta(\beta-1)^{1 / \beta-1}, \beta>1$. Suppose that for any given $t_{*} \geq t_{0}$, there exist intervals $I_{1}=\left[s_{1}, t_{1}\right], I_{2}=\left[s_{2}, t_{2}\right] \subset\left[t_{*}, \infty\right)$, such that $(a)$ - $(b)$ hold for all $i \in \mathbb{N}$.

If there exists $H \in \Phi\left(s_{k}, t_{k}\right)$ such that

$$
\begin{gathered}
\int_{s_{k}}^{t_{k}}\left\{\beta_{0}[q(t)]^{1 / \beta}|e(t)|^{1-1 / \beta} H^{2}(t)-r(t)\left|H^{\prime}(t)-\frac{p(t)}{2 r(t)} H(t)\right|^{2}\right\} \mathrm{d} t \\
+\sum_{s_{k} \leq \theta_{i}<t_{k}} \beta_{0} q_{i}^{1 / \beta}\left|e_{i}\right|^{1-1 / \beta} H^{2}\left(\theta_{i}\right)>0 \quad(k=1,2)
\end{gathered}
$$

then (4.62) is oscillatory.

Let $\beta=1$ in (4.62). Then we have the forced linear equation:

$$
\begin{gathered}
\left(r(t) x^{\prime}\right)^{\prime}+p(t) x^{\prime}+q(t) x=e(t), \quad t \neq \theta_{i}, \\
\Delta\left(r(t) x^{\prime}\right)+q_{i} x=e_{i}, \quad t=\theta_{i} .
\end{gathered}
$$

Corollary 4.21. Suppose that for any given $t_{*} \geq t_{0}$, there exist intervals $I_{1}=\left[s_{1}, t_{1}\right], I_{2}=\left[s_{2}, t_{2}\right] \subset$ $\left[t_{*}, \infty\right)$, such that $(a)-(b)$ hold for all $i \in \mathbb{N}$.

If there exists $H \in \Phi\left(s_{k}, t_{k}\right)$ such that

$$
\int_{s_{k}}^{t_{k}}\left\{q(t) H^{2}(t)-r(t)\left|H^{\prime}(t)-\frac{p(t)}{2 r(t)} H(t)\right|^{2}\right\} \mathrm{d} t+\sum_{s_{k} \leq \theta_{i}<t_{k}} q_{i} H^{2}\left(\theta_{i}\right)>0 \quad(k=1,2),
$$

then (4.64) is oscillatory.

Example 4.22. Consider

$$
\begin{gathered}
x^{\prime \prime}-(\sin t) x^{\prime}-(\cos t) x=e^{-\cos t} \sin t, \quad t \neq \frac{i \pi}{18}, \\
\Delta x^{\prime}+(\sigma \cos 3 t) x=-\tan t, \quad t=\frac{i \pi}{18},
\end{gathered}
$$

where $\sigma$ is a positive real number.

Let $H=\sin 6 t$. For any $T \geq 0$, choose $n \in \mathbb{N}$ sufficiently large so that $n \pi \geq T$ and set $I_{1}=[(2 n-5 / 6) \pi,(2 n-2 / 3) \pi]$ and $I_{2}=[(2 n+2 / 3) \pi,(2 n+5 / 6) \pi]$. Clearly, (a)-(b) are satisfied for all $n$. It is easy to see that for $j=2 n-5 / 6$ and $j=2 n+2 / 3$,

$$
\begin{aligned}
\int_{j \pi}^{(j+1 / 6) \pi} & {\left[-\cos t \sin ^{2} 6 t-\left(6 \cos 6 t+\frac{1}{2} \sin t \sin 6 t\right)^{2}\right] d t } \\
& +\sum_{j \pi \leq \theta_{i}<(j+1 / 6) \pi} \sigma \cos \left(3 \theta_{i}\right) \sin ^{2}\left(6 \theta_{i}\right) \\
= & \frac{18}{143}(-1+\sqrt{3})-\frac{289 \pi}{96}+\frac{3 \sigma}{8}(\sqrt{3}+1) .
\end{aligned}
$$


Thus (4.65) holds if

$$
\sigma>\frac{289 \pi}{72}(\sqrt{3}-1)-\frac{48}{143}(2-\sqrt{3})
$$

which by Corollary 4.21 is sufficient for oscillation of (4.66).

Note that if the impulses are removed, then (4.66) becomes nonoscillatory with a nonoscillatory solution $x(t)=t e^{-\cos t}$.

Finally we state a generalization of Theorem 4.17 for a class of more general type impulsive equations. Let $\alpha, \beta, q(t), e(t), \theta_{i},\left\{e_{i}\right\}$, and $\left\{q_{i}\right\}$ be as above, and consider

$$
\begin{gathered}
\left(r(t) \varphi_{\alpha}\left(x^{\prime}\right)\right)^{\prime}+p(t) \varphi_{\alpha}\left(x^{\prime}\right)+f(t, x)=e(t), \quad t \neq \theta_{i} \\
\Delta\left(r(t) \varphi_{\alpha}\left(x^{\prime}\right)\right)+g(t, x)=e_{i}, \quad t=\theta_{i}
\end{gathered}
$$

where the functions $f$ and $g$ satisfy

$$
\begin{aligned}
& s f(t, s) \geq q(t)|s|^{\beta+1} \quad \text { for } t \in I_{1} \cup I_{2}, s \in \mathbb{R}, \\
& s g\left(\theta_{i}, s\right) \geq q_{i}|s|^{\beta+1} \quad \text { for } \theta_{i} \in I_{1} \cup I_{2}, s \in \mathbb{R} .
\end{aligned}
$$

Theorem 4.23. In addition to conditions of Theorem 4.17, if (4.70) holds then (4.69) is oscillatory.

Example 4.24. Consider

$$
\begin{gathered}
\left(\left|x^{\prime}\right|^{-2 / 3} x^{\prime}\right)^{\prime}+(\cos 3 t)\left|x^{\prime}\right|^{-2 / 3} x^{\prime}+f(t, x)=\sin 3 t, \quad t \neq \frac{i \pi}{12} \\
\Delta\left(\left|x^{\prime}\right|^{-2 / 3} x^{\prime}\right)+g(t, x)=\cos ^{3} t \sin 3 t, \quad t=\frac{i \pi}{12}
\end{gathered}
$$

where

$$
\begin{gathered}
f(t, x)=\cos ^{2}\left(\frac{t}{2}\right)[2+\sin (x+t)]|x|^{1 / 2} x, \\
g(t, x)=\left[r+2 \cos ^{2}\left(\frac{x+t}{2}\right)\right]\left(\sec ^{3 / 2} t\right)|x|^{1 / 2} x, \quad(r>0) .
\end{gathered}
$$

Clearly if we take $I_{1}=[(2 n+5 / 3) \pi,(2 n+2) \pi]$ and $I_{2}=[(2 n+2) \pi,(2 n+7 / 3) \pi]$, then $(4.70)$ holds with $\beta=1 / 2, q(t)=\cos t$ and $q_{i}=\gamma \sec ^{3 / 2} t$. Further, we see that all conditions of Theorem 4.17 are satisfied if $H(t)=\sin ^{2} 3 t$ and $\gamma \geq 0.552$; see Example 4.18. Therefore we may deduce from Theorem 4.23 that (4.71) is oscillatory if $\gamma \geq 0.552$. 


\subsection{Oscillation of Second-Order Nonlinear Equations}

In this section, we first consider the second-order nonlinear impulsive differential equations of the following form:

$$
\begin{gathered}
x^{\prime \prime}(t)+f(t, x)=0, \quad t \neq t_{k}, \\
x\left(t_{k}^{+}\right)=g_{k}\left(x\left(t_{k}\right)\right), \quad x^{\prime}\left(t_{k}^{+}\right)=h_{k}\left(x^{\prime}\left(t_{k}\right)\right), \quad k \in \mathbb{N}, \\
x\left(t_{0}^{+}\right)=x_{0}, \quad x^{\prime}\left(t_{0}^{+}\right)=x_{0}^{\prime} .
\end{gathered}
$$

Assume that the following conditions hold.

(i) $f(t, x) \in C(J \times \mathbb{R}), x f(x)>0(x \neq 0)$, and $f(t, x) / \varphi(x) \geq p(t)(x \neq 0)$, where $p \in$ $C(J), p(t) \geq 0$, and $x \varphi(x)>0(x \neq 0), \varphi^{\prime}(x) \geq 0$.

(ii) $g_{k}, h_{k} \in C(\mathbb{R})$, and there exist positive numbers $a_{k}, a_{k}^{*}, b_{k}, b_{k}^{*}$ such that

$$
a_{k}^{*} \leq \frac{g_{k}(x)}{x} \leq a_{k}, \quad b_{k}^{*} \leq \frac{h_{k}(x)}{x} \leq b_{k} .
$$

In most of the investigations about oscillation of nonlinear impulsive differential equations, the following lemma is an important tool.

Lemma 4.25 (see [25]). Let $x(t)$ be a solution of (4.73). Suppose that there exists some $T \geq t_{0}$ such that $x(t)>0$ for $t \geq T$. If conditions ( $i$ ) and (ii) are satisfied, and

(iii) $\left(t_{1}-t_{0}\right)+\left(b_{1}^{*} / a_{1}\right)\left(t_{2}-t_{1}\right)+\left(b_{1}^{*} b_{2}^{*} / a_{1} a_{2}\right)\left(t_{3}-t_{2}\right)+\cdots+\left(b_{1}^{*} b_{2}^{*} \cdots b_{n}^{*} / a_{1} a_{2} \cdots a_{n}\right)\left(t_{n+1}-\right.$ $\left.t_{n}\right)+\cdots=+\infty$ holds, then $x^{\prime}\left(t_{k}^{+}\right) \geq 0$ and $x^{\prime}(t) \geq 0$ for $t \in\left(t_{k}, t_{k+1}\right]$, where $t_{k} \geq T$.

Theorems 4.26-4.32 are obtained in [25]. For some improvements and/or generalizations, see [32-35].

Theorem 4.26. Assume that conditions (i), (ii), and (iii) of Lemma 4.25 hold, and there exists a positive integer $k_{0}$, such that $a_{k}^{*} \geq 1$ for $k \geq k_{0}$. If

$$
\int_{t_{0}}^{t_{1}} p(t) d t+\frac{1}{b_{1}} \int_{t_{1}}^{t_{2}} p(t) d t+\frac{1}{b_{1} b_{2}} \int_{t_{2}}^{t_{3}} p(t) d t+\cdots+\frac{1}{b_{1} b_{2} \cdots b_{n}} \int_{t_{n}}^{t_{n+1}} p(t) d t+\cdots=\infty
$$

then every solution of (4.73) is oscillatory.

Proof. Without loss of generality, we can assume $k_{0}=1$. If (4.73) has a nonoscillatory solution $x(t)$, we might as well assume $x(t)>0, t \geq t_{0}$. From Lemma 4.25, $x^{\prime}(t) \geq 0$ for $t \in\left(t_{k}, t_{k+1}\right]$, where $k \in \mathbb{N}$. Let

$$
u(t)=\frac{x^{\prime}(t)}{\varphi(x(t))}
$$


Then, $u\left(t_{k}^{+}\right) \geq 0(k \in \mathbb{N}), u(t) \geq 0\left(t \geq t_{0}\right)$. Using condition (i) in (4.73), we get for $t \neq t_{k}$,

$$
u^{\prime}(t)=-\frac{f(t, x(t))}{\varphi(x(t))}-\left[\frac{x^{\prime}(t)}{\varphi(x(t))}\right]^{2} \varphi^{\prime}(x(t)) \leq-p(t)
$$

Using condition (ii) and $a_{k}^{*} \geq 1, \varphi^{\prime}(x) \geq 0$ yield

$$
u\left(t_{k}^{+}\right)=\frac{x^{\prime}\left(t_{k}^{+}\right)}{\varphi\left(x\left(t_{k}^{+}\right)\right)} \leq b_{k} u\left(t_{k}\right), \quad k \in \mathbb{N}
$$

From the above inequalities, we have

$$
u\left(s_{1}\right) \leq u(s)-\int_{s}^{s_{1}} p(t) d t
$$

where $t_{0}<s<s_{1}<t_{1}$. Taking $s \rightarrow t_{0}^{+}$and $s_{1} \rightarrow t_{1}$, we get

$$
u\left(t_{1}^{+}\right) \leq b_{1} u\left(t_{1}\right) \leq b_{1}\left[u\left(t_{0}^{+}\right)-\int_{t_{0}}^{t_{1}} p(t) d t\right] \leq b_{1} u\left(t_{0}^{+}\right)-b_{1} \int_{t_{0}}^{t_{1}} p(t) d t
$$

By induction, for any natural number $n$, we obtain

$$
\begin{aligned}
u\left(t_{n}^{+}\right) \leq b_{1} b_{2} \cdots b_{n}\left[u\left(t_{0}^{+}\right)-\int_{t_{0}}^{t_{1}} p(t) d t-\frac{1}{b_{1}} \int_{t_{1}}^{t_{2}} p(t) d t\right. & \\
& \left.\quad \cdots-\frac{1}{b_{1} b_{2} \cdots b_{n-2}} \int_{t_{n-2}}^{t_{n-1}} p(t) d t-\frac{1}{b_{1} b_{2} \cdots b_{n-1}} \int_{t_{n-1}}^{t_{n}} p(t) d t\right] .
\end{aligned}
$$

Since, $u\left(t_{k}^{+}\right) \geq 0(k \in \mathbb{N})$, above inequality and the hypothesis lead to a contradiction. So, every solution of (4.73) oscillatory.

From Theorem 4.26, the following corollary is immediate.

Corollary 4.27. Assume that conditions (i)-(iii) of Lemma 4.25 hold and there exists a positive integer $k_{0}$ such that $a_{k}^{*} \geq 1, b_{k} \leq 1$ for $k \geq k_{0}$. If

$$
\int^{\infty} p(t) d t=\infty
$$

then every solution of (4.73) is oscillatory.

The proof of the following theorem is similar to that of Theorem 4.26. 
Theorem 4.28. Assume that conditions (i), (ii), and (iii) of Lemma 4.25 hold and $\varphi(a b) \geq$ $\varphi(a) \varphi(b)$ for any $a b>0$. If

$$
\begin{gathered}
\int_{t_{0}}^{t_{1}} p(t) d t+\frac{\varphi\left(a_{1}^{*}\right)}{b_{1}} \int_{t_{1}}^{t_{2}} p(t) d t+\frac{\varphi\left(a_{1}^{*}\right) \varphi\left(a_{2}^{*}\right)}{b_{1} b_{2}} \int_{t_{2}}^{t_{3}} p(t) d t+\cdots \\
+\frac{\varphi\left(a_{1}^{*}\right) \varphi\left(a_{2}^{*}\right) \cdots \varphi\left(a_{n}^{*}\right)}{b_{1} b_{2} \cdots b_{n}} \int_{t_{n}}^{t_{n+1}} p(t) d t+\cdots=\infty
\end{gathered}
$$

then every solution of (4.73) is oscillatory.

Corollary 4.29. Assume that conditions (i), (ii), and (iii) of Lemma 4.25 hold and $\varphi(a b) \geq$ $\varphi(a) \varphi(b)$ for any $a b>0$. Suppose that there exist a positive integer $k_{0}$ and a constant $\alpha>0$ such that

$$
\frac{\varphi\left(a_{k}^{*}\right)}{b_{k}} \geq\left(\frac{t_{k+1}}{t_{k}}\right)^{\alpha} \text { for } k \geq k_{0} .
$$

If $\int^{\infty} t^{\alpha} p(t) d t=\infty$, then every solution of (4.73) is oscillatory.

Example 4.30. Consider the superlinear equation:

$$
\begin{gathered}
x^{\prime \prime}+\left(\frac{1}{t^{3}}\right) x^{2 n-1}=0, \quad t \geq \frac{1}{2}, \quad t \neq k, \\
x\left(k^{+}\right)=\left(\frac{k+1}{k}\right) x(k), \quad x^{\prime}\left(k^{+}\right)=x^{\prime}(k), \quad k \in \mathbb{N}, \\
x\left(\frac{1}{2}\right)=x_{0}, \quad x^{\prime}\left(\frac{1}{2}\right)=x_{0}^{\prime},
\end{gathered}
$$

where $n>1$ is a natural number. Since $a_{k}=a_{k}^{*}=(k+1) / k, b_{k}=b_{k}^{*}=1, p(t)=1 / t^{3}, t_{k}=k$, and $\varphi(x)=x^{2 n-1}$. It is easy to see that conditions (i), (ii), and (iii) are satisfied. Moreover

$$
\begin{aligned}
& \frac{\varphi\left(a_{k}^{*}\right)}{b_{k}}=\left(\frac{k+1}{k}\right)^{2 n-1} \geq\left(\frac{t_{k+1}}{t_{k}}\right)^{3}, \\
& \int^{\infty} t^{\alpha} p(t) d t=\int^{\infty} t^{3}\left(\frac{1}{t^{3}}\right) d t=\infty .
\end{aligned}
$$

Hence, by Corollary 4.29, we find that every solution of (4.85) is oscillatory. On the other hand, by Theorem 2.6, it follows that (4.85) without impulses is nonoscillatory. 
Theorem 4.31. Assume that conditions (i), (ii), and (iii) of Lemma 4.25 hold, and there exists a positive integer $k_{0}$ such that $a_{k}^{*} \geq 1$ for $k \geq k_{0}$. If

$$
\begin{gathered}
\int_{ \pm \epsilon}^{ \pm \infty} \frac{d u}{\varphi(u)}<\infty, \text { for some } \epsilon>0 \\
\sum_{k=0}^{\infty} \int_{t_{k}}^{t_{k+1}}\left[\int_{s}^{t_{k+1}} p(t) d t+\frac{1}{b_{k+1}} \int_{t_{k+1}}^{t_{k+2}} p(t) d t+\frac{1}{b_{k+1} b_{k+2}} \int_{t_{k+2}}^{t_{k+3}} p(t) d t+\cdots\right. \\
\left.+\frac{1}{b_{k+1} b_{k+2} \cdots b_{k+n}} \int_{t_{k+n}}^{t_{k+n+1}} p(t) d t+\cdots\right] d s=\infty
\end{gathered}
$$

then every solution of (4.73) is oscillatory.

Proof. Without loss of generality, let $k_{0}=1$. If (4.1) has a nonoscillatory solution $x(t)$, assume $x(t)>0\left(t \geq t_{0}\right)$. By Lemma 4.25, $x^{\prime}\left(t_{k}^{+}\right) \geq 0(k \in \mathbb{N}), x^{\prime}(t) \geq 0\left(t \geq t_{0}\right)$. Since $a_{k}^{*} \geq 1(k \in \mathbb{N})$, we have

$$
x\left(t_{0}^{+}\right) \leq x\left(t_{1}\right) \leq x\left(t_{1}^{+}\right) \leq x\left(t_{2}\right) \leq x\left(t_{2}^{+}\right) \leq \cdots
$$

It is easy to see that $x(t)$ is monotonically nondecreasing in $J$. Now (4.73) yields

$$
x^{\prime \prime}(t)=-f(t, x(t)) \leq-p(t) \varphi(x(t)), \quad t \neq t_{k} .
$$

Hence, from the above inequality and condition (ii), we find that

$$
x^{\prime}\left(t_{0}^{+}\right) \geq \int_{t_{0}}^{t_{1}} p(t) \varphi(x(t)) d t+x^{\prime}\left(t_{1}\right) \geq \int_{t_{0}}^{t_{1}} p(t) \varphi(x(t)) d t+\frac{x^{\prime}\left(t_{1}^{+}\right)}{b_{1}} .
$$

Generally, for any natural number $n$, we have

$$
\begin{aligned}
x^{\prime}\left(t_{n}^{+}\right) & \geq \int_{t_{n}}^{t_{n+1}} p(t) \varphi(x(t)) d t+x^{\prime}\left(t_{n+1}\right) \\
& \geq \int_{t_{n}}^{t_{n+1}} p(t) \varphi(x(t)) d t+\frac{x^{\prime}\left(t_{n+1}^{+}\right)}{b_{n+1}} .
\end{aligned}
$$

By (4.89) and (4.91), noting $x\left(t_{k}^{+}\right) \geq 0(k \in \mathbb{N})$ and $b_{i}>0(i \in \mathbb{N})$, for any natural number $n$, we obtain

$$
\begin{aligned}
x^{\prime}(s) \geq & \int_{s}^{t_{k+1}} p(t) \varphi(x(t)) d t+\frac{1}{b_{k+1}} \int_{t_{k+1}}^{t_{k+2}} p(t) \varphi(x(t)) d t \\
& +\cdots+\frac{1}{b_{k+1} b_{k+2} \cdots b_{k+n}} \int_{t_{k+n}}^{t_{k+n+1}} p(t) \varphi(x(t)) d t+\cdots
\end{aligned}
$$


Note that $x \varphi(x)>0(x \neq 0)$ and $\varphi(x)$ is nondecreasing. Dividing the above inequality by $\varphi(x(s))$ and then integrating from $t_{k}$ to $t_{k+1}$, we get

$$
\begin{aligned}
& \int_{t_{k}}^{t_{k+1}}\left[\int_{s}^{t_{k+1}} p(t) d t+\frac{1}{b_{k+1}} \int_{t_{k+1}}^{t_{k+2}} p(t) d t+\cdots+\frac{1}{b_{k+1} b_{\mathrm{k}+2} \cdots b_{k+n}} \int_{t_{k+n}}^{t_{k+n+1}} p(t) d t+\cdots\right] d s \\
& \quad \leq \int_{t_{k}}^{t_{k+1}} \frac{x^{\prime}(s)}{\varphi(x(s))} d s=\int_{x\left(t_{k}^{+}\right)}^{x\left(t_{k+1}\right)} \frac{1}{\varphi(u)} d u .
\end{aligned}
$$

Since (4.88) holds, the above inequality yields

$$
\begin{aligned}
& \sum_{k=0}^{\infty} \int_{t_{k}}^{t_{k+1}}\left[\int_{s}^{t_{k+1}} p(t) d t+\frac{1}{b_{k+1}} \int_{t_{k+1}}^{t_{k+2}} p(t) d t\right. \\
& \left.\quad+\cdots+\frac{1}{b_{k+1} b_{k+2} \cdots b_{k+n}} \int_{t_{k+n}}^{t_{k+n+1}} p(t) d t+\cdots\right] d s \\
& \leq \sum_{k=0}^{\infty} \int_{x\left(t_{k}^{+}\right)}^{x\left(t_{k+1}\right)} \frac{1}{\varphi(u)} d u \leq \int_{x\left(t_{0}^{+}\right)}^{\infty} \frac{1}{\varphi(u)} d u .
\end{aligned}
$$

The above inequality and the hypotheses lead to a contradiction. So, every solution of (4.73) is oscillatory.

The proof of the following theorem is similar to that of Theorem 4.31.

Theorem 4.32. Assume that conditions (i), (ii), and (iii) of Lemma 4.25 hold, and there exists a positive integer $k_{0}$ such that $a_{k}^{*} \geq 1$ for $k \geq k_{0}$. Suppose that $\varphi(a b) \geq \varphi(a) \varphi(b)$ for any $a b>0$ and

$$
\begin{aligned}
& \int_{ \pm \epsilon}^{ \pm \infty} \frac{d u}{\varphi(u)}<\infty, \text { for some } \epsilon>0 \\
& \sum_{k=0}^{\infty} \int_{t_{k}}^{t_{k+1}}\left[\int_{s}^{t_{k+1}} p(t) d t+\frac{\varphi\left(a_{k+1}^{*}\right)}{b_{k+1}} \int_{t_{k+1}}^{t_{k+2}} p(t) d t\right. \\
& +\frac{\varphi\left(a_{k+1}^{*}\right) \varphi\left(a_{k+2}^{*}\right)}{b_{k+1} b_{k+1}} \int_{t_{k+2}}^{t_{k+3}} p(t) d t+\cdots \\
& \left.+\frac{\varphi\left(a_{k+1}^{*}\right) \varphi\left(a_{k+2}^{*}\right) \cdots \varphi\left(a_{k+n}^{*}\right)}{b_{k+1} b_{k+1} \cdots b_{k+n}} \int_{t_{k+n}}^{t_{k+n+1}} p(t) d t+\cdots\right] d s=\infty .
\end{aligned}
$$

Then, every solution of (4.73) is oscillatory. 
In [36], the author studied the second-order nonlinear impulsive differential equations of the following form:

$$
\begin{gathered}
\left(r(t) \psi(x) \varphi_{\alpha}\left(x^{\prime}\right)\right)^{\prime}+p(t) \psi(x) \varphi_{\alpha}\left(x^{\prime}\right)+q(t) f(x)=0, \quad t \neq \theta_{i} \\
\Delta\left(r \psi(x) \varphi_{\alpha}\left(x^{\prime}\right)\right)+q_{i} f(x)=0, \quad t=\theta_{i}
\end{gathered}
$$

where $\varphi_{\alpha}(s):=|s|^{\alpha-1} s$ and $\Delta z(t):=z\left(t^{+}\right)-z\left(\mathrm{t}^{-}\right), z\left(t^{ \pm}\right)=\lim _{\tau \rightarrow t^{ \pm}} z(\tau)$.

Sufficient conditions are obtained for oscillation of (4.96) by using integral averaging technique. In particular the Philos type oscillation criteria are extended to impulsive differential equations.

It is assumed that

(i) $\alpha>0$ is a constant;

(ii) $\left\{\theta_{i}\right\}$ is a strictly increasing unbounded sequence of real numbers; $\left\{q_{i}\right\}$ is a real sequence;

(iii) $r, p, q \in \operatorname{PLC}(J), r(t)>0$;

(iv) $\psi \in C(\mathbb{R}), f \in C^{1}(\mathbb{R})$ with $\psi(s)>0, s f(s)>0$ for $s \neq 0$ and

$$
\frac{f^{\prime}(s)}{\left(\psi(s)|f(s)|^{\alpha-1}\right)^{1 / \alpha}} \geq K_{\alpha}
$$

is satisfied; $K_{\alpha}>0$ is a constant.

In order to prove the results the following well-known inequality is needed [26].

Lemma 4.33. If $A, B$ are nonnegative numbers, then

$$
A^{\gamma}-\gamma A B^{\gamma-1}+(\gamma-1) B^{\gamma} \geq 0, \quad r>1
$$

and the equality holds if and only if $A=B$.

The following theorem is one of the main results of this study.

Theorem 4.34 (see [36]). Let $D_{0}=\left\{(t, s): t>s>t_{0}\right\}$ and $D=\left\{(t, s): t \geq s \geq t_{0}\right\}$. Assume that $H(t, s) \in C^{1}(D,(0, \infty)), h(t, s) \in C\left(D_{0}, \mathbb{R}\right)$ satisfy the following conditions.

(i) $H(t, t)=0$ for $t \geq t_{0}$ and $H(t, s)>0$ on $D_{0}$.

(ii) $H$ has a continuous and nonpositive partial derivative on $D_{0}$ with respect to the second variable.

(iii) One has

$$
-\frac{\partial H}{\partial s}(t, s)+\frac{p(s)}{r(s)} H(t, s)=h(t, s) H^{\frac{\alpha}{\alpha+1}}(t, s), \quad(t, s) \in D_{0} .
$$




$$
\limsup _{t \rightarrow \infty} \frac{1}{H\left(t, t_{0}\right)}\left\{\int_{t_{0}}^{t}\left[H(t, s) q(s)-\Gamma_{\alpha} r(s)|h(t, s)|^{\alpha+1}\right] d s+\sum_{t_{0} \leq \theta_{i}<t} H\left(t, \theta_{i}\right) q_{i}\right\}=\infty,
$$

where $\Gamma_{\alpha}=(\alpha+1)^{-(\alpha+1)} \alpha^{\alpha} K_{\alpha}^{-\alpha}$, then (4.96) is oscillatory.

Proof. Let $x(t)$ be a nonoscillatory solution of (4.96). We assume that $x(t) \neq 0$ on $[T, \infty)$ for some sufficiently large $T \geq t_{0}$. Define

$$
w(t):=\frac{r(t) \psi(x) \varphi_{\alpha}\left(x^{\prime}\right)}{f(x)}, \quad t \geq T
$$

Differentiating (4.101) and making use of (4.96) and (4.97), we obtain

$$
\begin{gathered}
w^{\prime}(t) \leq-\frac{p(t)}{r(t)} w(t)-q(t)-K_{\alpha} \frac{|w(t)|^{1+1 / \alpha}}{r^{1 / \alpha}(t)}, \quad t \neq \theta_{i} \\
\Delta w(t)=-q_{i}, \quad t=\theta_{i} .
\end{gathered}
$$

Replacing $t$ by $s$ in (4.102) and multiplying the resulting equation by $H(t, s)$ and integrating from $T$ to $t$, we get

$$
\begin{aligned}
\int_{T}^{t} H(t, s) q(s) d s \leq & -\int_{T}^{t} \frac{p(s)}{r(s)} H(t, s) w(s) d s \\
& -K_{\alpha} \int_{T}^{t} H(t, s) \frac{|w(s)|^{1+1 / \alpha}}{r^{1 / \alpha}(s)} d s-\int_{T}^{t} H(t, s) w^{\prime}(s) d s .
\end{aligned}
$$

Integrating by parts and using (4.103), we find

$$
\begin{aligned}
\int_{T}^{t} H(t, s) w^{\prime}(s) d s & =\int_{T}^{t}\left[\frac{\partial}{\partial s}\{H(t, s) w(s)\}-w(s) \frac{\partial H}{\partial s}(t, s)\right] d s \\
& =-H(t, T) w(T)+\sum_{T \leq \theta_{i}<t} H\left(t, \theta_{i}\right) q_{i}-\int_{T}^{t} w(s) \frac{\partial H}{\partial s}(t, s) d s .
\end{aligned}
$$

Combining (4.104) and (4.105), we obtain

$$
\begin{aligned}
& \int_{T}^{t} H(t, s) q(s) d s+\sum_{T \leq \theta_{i}<t} H\left(t, \theta_{i}\right) q_{i} \\
& \quad \leq H(t, T) w(T)-\int_{T}^{t}\left[K_{\alpha} H(t, s) \frac{|w(s)|^{1+1 / \alpha}}{r^{1 / \alpha}(s)}-|h(t, s)| H^{\alpha /(\alpha+1)}(t, s)|w(s)|\right] d s .
\end{aligned}
$$


Using inequality (4.98) with $\gamma=1+1 / \alpha$,

$$
A=\frac{\left(K_{\alpha} H\right)^{\alpha /(\alpha+1)}|w|}{r^{1 /(\alpha+1)}}, \quad B=\left(\frac{\alpha}{\alpha+1}\right)^{\alpha}\left(\frac{r}{K_{\alpha}^{\alpha}}\right)^{\alpha /(\alpha+1)}|h|^{\alpha},
$$

we find

$$
K_{\alpha} H(t, s) \frac{|w(s)|^{1+1 / \alpha}}{r^{1 / \alpha}(s)}-|h(t, s)| H^{\alpha /(\alpha+1)}(t, s)|w(s)| \geq-\Gamma_{\alpha} r(s)|h(t, s)|^{\alpha+1} .
$$

From (4.106) and (4.108), we obtain

$$
\begin{gathered}
\int_{T}^{t}\left[H(t, s) q(s)-\Gamma_{\alpha} r(s)|h(t, s)|^{\alpha+1}\right] d s+\sum_{T \leq \theta_{i}<t} H\left(t, \theta_{i}\right) q_{i} \\
\leq H(t, T) w(T) \leq H(t, T)|w(T)| \leq H\left(t, t_{0}\right)|w(T)|
\end{gathered}
$$

for all $t>T \geq t_{0}$. In the above inequality we choose $T=T_{0}$, to get

$$
\begin{aligned}
\int_{t_{0}}^{t}\left[H(t, s) q(s)-\Gamma_{\alpha} r(s)|h(t, s)|^{\alpha+1}\right] d s+\sum_{t_{0} \leq \theta_{i}<t} H\left(t, \theta_{i}\right) q_{i} \\
\leq H\left(t, t_{0}\right)\left\{\int_{t_{0}}^{T_{0}}|q(s)| d s+\sum_{t_{0} \leq \theta_{i}<T_{0}}\left|q_{i}\right|\right\}+H\left(t, t_{0}\right)\left|w\left(T_{0}\right)\right| .
\end{aligned}
$$

Thus, it follows that

$$
\begin{aligned}
& \limsup _{t \rightarrow \infty} \frac{1}{H\left(t, t_{0}\right)}\left\{\int_{t_{0}}^{t}\left[H(t, s) q(s)-\Gamma_{\alpha} r(s)|h(t, s)|^{\alpha+1}\right] d s+\sum_{t_{0} \leq \theta_{i}<t} H\left(t, \theta_{i}\right) q_{i}\right\} \\
& \leq \int_{t_{0}}^{T_{0}}|q(s)| d s+\sum_{t_{0} \leq \theta_{i}<T_{0}}\left|q_{i}\right|+\left|w\left(T_{0}\right)\right|<\infty,
\end{aligned}
$$

which contradicts (4.100). This completes the proof.

As a corollary to Theorem 4.34, we have the following result.

Corollary 4.35. Let condition (4.100) in Theorem 4.34 be replaced by

$$
\begin{gathered}
\limsup _{t \rightarrow \infty} \frac{1}{H\left(t, t_{0}\right)}\left\{\int_{t_{0}}^{t} H(t, s) q(s) d s+\sum_{t_{0} \leq \theta_{i}<t} H\left(t, \theta_{i}\right) q_{i}\right\}=\infty, \\
\limsup _{t \rightarrow \infty} \frac{1}{H\left(t, t_{0}\right)} \int_{t_{0}}^{t} r(s)|h(t, s)|^{\alpha+1} d s<\infty,
\end{gathered}
$$

then (4.96) is oscillatory. 
Note that in the special case of half-linear equations, for $\psi(s) \equiv 1$ and $f(s)=\varphi_{\alpha}(s)$, the condition (4.97) is satisfied with $K_{\alpha}=\alpha$.

The proof of the following theorem can be accomplished by using the method developed for the nonimpulsive case and similar arguments employed in the proof of Theorem 4.34 .

Theorem 4.36 (see [36]). Let the functions $H$ and $h$ be defined as in Theorem 4.34. Moreover, Suppose that

$$
\begin{gathered}
0<\inf _{s \geq t_{0}}\left\{\liminf _{t \rightarrow \infty} \frac{H(t, s)}{H\left(t, t_{0}\right)}\right\} \leq \infty, \\
\limsup _{t \rightarrow \infty} \frac{1}{H\left(t, t_{0}\right)} \int_{t_{0}}^{t} r(s)|h(t, s)|^{\alpha+1} d s<\infty .
\end{gathered}
$$

If there exists a function $A \in C(J, \mathbb{R})$ such that

$$
\limsup _{t \rightarrow \infty} \int_{t_{0}}^{t} \frac{A_{+}^{1+1 / \alpha}(s)}{r^{1 / \alpha}(s)} d s=\infty
$$

and for every $T \geq t_{0}$

$$
\limsup _{t \rightarrow \infty} \frac{1}{H(t, T)}\left\{\int_{T}^{t}\left[H(t, s) q(s)-\Gamma_{\alpha} r(s)|h(t, s)|^{\alpha+1}\right] d s+\sum_{T \leq \theta_{i}<t} H\left(t, \theta_{i}\right) q_{i}\right\} \geq A(T),
$$

where $A_{+}(s)=\max \{A(s), 0\}$, then (4.96) is oscillatory.

\subsection{Higher-Order Nonlinear Equations}

There are only a very few works concerning the oscillation of higher-order nonlinear impulsive differential equations [37-40].

In [37] authors considered even order impulsive differential equations of the following form

$$
\begin{aligned}
x^{(2 n)}+f(t, x) & =0, \quad t \neq t_{k}, \\
x^{(i)}\left(t_{k}^{+}\right)=g_{k(i)}\left(x^{(i)}\left(t_{k}\right)\right), \quad i & =0,1, \ldots, 2 n-1, \quad k \in \mathbb{N}, \\
x^{(i)}\left(t_{0}^{+}\right) & =x_{0}^{(i)},
\end{aligned}
$$


where

$$
\begin{aligned}
x^{(i)}\left(t_{k}\right) & =\lim _{h \rightarrow 0^{-}} \frac{x^{(i-1)}\left(t_{k}+h\right)-x^{(i-1)}\left(t_{k}\right)}{h}, \\
x^{(i)}\left(t_{k}^{+}\right) & =\lim _{h \rightarrow 0^{+}} \frac{x^{(i-1)}\left(t_{k}+h\right)-x^{(i-1)}\left(t_{k}^{+}\right)}{h} .
\end{aligned}
$$

Let the following conditions hold.

(A) $f(t, x) \in C(J \times \mathbb{R}) ; x f(t, x)>0$ for $x \neq 0 ; f(t, x) / \varphi(x) \geq p(t)$ for $x \neq 0$, where $p(t)$ is positive and continuous on $J ; x \varphi(x)>0$ for $x \neq 0 ; \varphi^{\prime}(x) \geq 0$.

(B) $g_{k(i)} \in C(\mathbb{R})$, and there exist positive numbers $a_{k}^{(i)}, b_{k}^{(i)}, i=0,1, \ldots, 2 n-1$ such that

$$
\begin{gathered}
a_{k}^{(i)} \leq \frac{g_{k(i)}(x)}{x} \leq b_{k(i)}, \\
\left(t_{1}-t_{0}\right)+\frac{a_{1}^{(i)}}{b_{1}^{(i-1)}}\left(t_{2}-t_{1}\right)+\frac{a_{1}^{(i)} a_{2}^{(i)}}{b_{1}^{(i-1)} b_{2}^{(i-1)}}\left(t_{3}-t_{2}\right)+\cdots+\frac{a_{1}^{(i)} a_{2}^{(i)} \cdots a_{m}^{(i)}}{b_{1}^{(i-1)} b_{2}^{(i-1)} \cdots b_{m}^{(i-1)}}\left(t_{m+1}-t_{m}\right)+\cdots=\infty .
\end{gathered}
$$

A function $x:\left[t_{0}, t_{0}+\alpha\right) \rightarrow \mathbb{R}, t_{0}>0, \alpha>0$ is said to be a solution of (4.116), if (i) $x^{(i)}\left(t_{0}^{+}\right)=x_{0}^{(i)}, i=0,1, \ldots, 2 n-1$; (ii) for $t \in\left[t_{0}, t_{0}+\alpha\right)$ and $t \neq t_{k}, x(t)$ satisfies $x^{(2 n)}(t)+$ $f(t, x(t))=0$; (iii) $x^{(i)}(t)$ is left continuous on $t_{k} \in\left[t_{0}, t_{0}+\alpha\right)$, and $x^{(i)}\left(t_{k}^{+}\right)=g_{k(i)} x^{(i)}\left(t_{k}\right), i=$ $0,1, \ldots, 2 n-1$.

The first two theorems can be considered as modifications of Theorems 3.43 and 4.26, respectively.

Theorem 4.37 (see [37]). If conditions $(A)$ and $(B)$ hold, $b_{k}^{(i)} \leq 1, a_{k}^{(0)} \geq 1, b_{k}^{(0)} \geq 1(i=$ $1,2, \ldots, 2 n-1 ; k=1,2, \ldots)$, and if

$$
\int^{\infty} t^{2 n-1} p(t) d t=\infty
$$

then every bounded solution of (4.116) is oscillatory.

Theorem 4.38 (see [37]). If conditions $(A)$ and $(B)$ hold, $a_{k}^{(0)} \geq 1$ and

$$
\begin{aligned}
\int_{t_{0}}^{t_{1}} p(t) d t+\frac{1}{b_{1}^{(2 n-1)}} \int_{t_{1}}^{t_{2}} p(t) d t+\frac{1}{b_{1}^{(2 n-1)} b_{2}^{(2 n-1)}} \int_{t_{2}}^{t_{3}} p(t) d t \\
+\cdots+\frac{1}{b_{1}^{(2 n-1)} b_{2}^{(2 n-1)} \cdots b_{m}^{(2 n-1)}} \int_{t_{m}}^{t_{m+1}} p(t) d t+\cdots=\infty
\end{aligned}
$$

then every solution of (4.116) is oscillatory. 
Theorem 4.39 (see [37]). If conditions ( $A$ ) and (B) hold, $\prod_{k=1}^{m} a_{k}^{(0)}>b>0 \quad(m=1,2, \ldots), b_{k}^{(2 n-1)} \leq$ 1 , and for any $\delta>0$,

$$
\mid \int_{\delta \leq|x|<\infty}^{\infty} \inf _{\delta(t, x) d t \mid=\infty}
$$

then every solution of (4.116) is oscillatory.

Corollary 4.40. Assume that conditions $(A)$ and (B) hold, and that $a_{k}^{(0)} \geq 1, b_{k}^{(2 n-1)} \leq 1$. If

$$
\int^{\infty} p(t) d t=\infty
$$

then every solution of (4.116) is oscillatory.

Corollary 4.41. Assume that conditions $(A)$ and $(B)$ hold, and that there exists a positive number $\alpha>0$, such that $a_{k}^{(0)} \geq 1,1 / b_{k}^{(2 n-1)} \geq\left(t_{k+1} / t_{k}\right)^{\alpha}$. If

$$
\int^{\infty} t^{\alpha} p(t) d t=\infty
$$

then every solution of (4.116) is oscillatory.

Example 4.42. Consider the impulsive differential equation:

$$
\begin{gathered}
x^{(2 n)}+\frac{1}{4 t} x^{3}=0, \quad t \geq \frac{1}{2}, \quad t \neq k, \quad k \in \mathbb{N}, \\
x\left(k^{+}\right)=\frac{k+1}{k} x(k), \quad x^{(i)}\left(k^{+}\right)=x^{(i)}(k), \quad i=1, \ldots, 2 n-1, \\
x\left(\frac{1}{2}\right)=x_{0}, \quad x^{(i)}\left(\frac{1}{2}\right)=x_{0}^{(i)},
\end{gathered}
$$

where $a_{k}^{(0)}=b_{k}^{(0)}=(k+1) / k>1, a_{k}^{(i)}=b_{k}^{(i)}=1, i=1,2, \ldots, 2 n-1, p(t)=1 / 4 t, \varphi(x)=x^{3}$, $f(t, x)=(1 / 4 t) x^{3}, t_{k}=k, t_{0}=1 / 2$.

It is easy to verify conditions of Theorem 4.38. So every solution of (4.124) is oscillatory.

Example 4.43. Consider the impulsive differential equation:

$$
\begin{gathered}
x^{(2 n)}+\frac{1}{t^{2}} x^{1 / 3}=0, \quad t \geq \frac{1}{2}, t \neq k, \quad k \in \mathbb{N}, \\
x\left(k^{+}\right)=x(k), \quad x^{(i)}\left(k^{+}\right)=\frac{k}{k+1} x^{(i)}(k), \quad i=1, \ldots, 2 n-1, \\
x\left(\frac{1}{2}\right)=x_{0}, \quad x^{(i)}\left(\frac{1}{2}\right)=x_{0}^{(i)},
\end{gathered}
$$


where $a_{k}^{(0)}=b_{k}^{(0)}=1, a_{k}^{(i)}=b_{k}^{(i)}=k /(k+1), i=1,2, \ldots, 2 n-1, p(t)=1 / t^{2}, t_{k}=k, \varphi(x)=x^{1 / 3}$, $f(t, x(t))=\left(1 / t^{2}\right) x^{1 / 3}(t), t_{0}=1 / 2$.

In this case, it can be show that conditions of Corollary 4.41 are satisfied. Thus, every solution of (4.125) is oscillatory.

In [40], the authors considered the impulsive differential equations with piecewise constant argument of the following form:

$$
\begin{gathered}
x^{(m)}(t)+a(t)\left(x^{(\gamma)}([t])\right)^{2 p}+e(t) \operatorname{sign} x(t)=0, \quad t \neq n, t \geq 0, n \in \mathbb{Z}^{+}, \\
x^{(j)}(n)=\beta_{n}^{(j)} x^{(j)}\left(n^{-}\right), \\
x^{(j)}\left(\left[t_{0}\right]\right)=x_{0}^{(j)},
\end{gathered}
$$

where $\mathbb{Z}^{+}$is the set of all positive integers, $m>1, \gamma$ and $p$ are given positive constants, [-] denotes the set of maximum integers, and $x_{0}^{(j)}, \beta_{n}^{(j)}>0$ for all $n, j$. It is assumed that

(i) $a, e \in C\left(J, \mathbb{R}^{+}\right)$;

(ii) for any $n>0$ and all $i=1,2, \ldots, m$,

$$
\frac{\beta_{n+1}^{(m-i)}}{\beta_{n+1}^{(m-i)}}+\frac{\beta_{n+2}^{(m-i)} \beta_{n+1}^{(m-i)}}{\beta_{n+2}^{(m-i-1)} \beta_{n+1}^{(m-1-1)}}+\cdots+\frac{\prod_{s=1}^{j-1} \beta_{n+1}^{(m-i)}}{\prod_{s=1}^{j-1} \beta_{n+s}^{(m-i-1)}} \longrightarrow \infty, \quad j \longrightarrow \infty
$$

(iii) there exists $C>0$ such that

$$
\inf _{t \geq 0} \frac{e(t)}{a(t)} \geq C
$$

Theorem 4.44 (see [40]). Assume that conditions (i), (ii), and (iii) hold. Moreover, suppose that for any $n>0$, there exists $a j>0$ such that

$$
\begin{gathered}
\int_{n}^{n+1} a(s) d s+\left(\beta_{n+1}^{(m-1)}\right)^{-1} \int_{n+1}^{n+2} \frac{a(s)}{A_{n+1}^{1}} d s+\left(\beta_{n+1}^{(m-1)} \beta_{n+2}^{(m-1)}\right)^{-1} \int_{n+2}^{n+3} \frac{a(s)}{A_{n+2}^{2}} d s+\cdots \\
+\prod_{s=1}^{j-1}\left(\beta_{n+s}^{(m-1)}\right)^{-1} \int_{n+j-1}^{n+j} \frac{a(s)}{A_{n+j-1}^{j-1}} d s \longrightarrow \infty, \quad j \longrightarrow \infty,
\end{gathered}
$$

where $A_{n}^{k}=n ! / k !$. Then every solution of (4.126) is oscillatory. 
Theorem 4.45 (see [40]). Assume that conditions (i) and (ii) hold. Moreover, suppose that for any $n>0$, there exists a $j>0$ such that

$$
\begin{gathered}
\prod_{s=1}^{n}\left(\beta_{s}^{(m-1)}\right)^{-1} \int_{n}^{n+1} e(s) d s+\prod_{s=1}^{n+1}\left(\beta_{s}^{(m-1)}\right)^{-1} \int_{n+1}^{n+2} e(s) d s+\cdots \\
+\prod_{s=1}^{n+j-1}\left(\beta_{s}^{(m-1)}\right)^{-1} \int_{n+j-1}^{n+j} e(s) d s>x_{0}^{(m-1)}
\end{gathered}
$$

Then every solution of (4.126) is oscillatory.

Example 4.46. Consider the impulsive differential equation:

$$
\begin{gathered}
x^{(m)}+\ln (1+t)\left(x^{(m-1)}([t])\right)^{2}+e^{-t} \operatorname{sign} x(t)=0, \quad t \neq n, t \geq 0, n \in \mathbb{Z}^{+}, \\
x^{(j)}(n)=\left(\frac{1}{3}\right) x^{(j)}\left(n^{-}\right), \\
x^{(j)}(0)=2 .
\end{gathered}
$$

It is easy to verify that conditions of Theorem 4.45 are all satisfied. Therefore every solution of (4.131) is oscillatory.

\section{Acknowledgments}

This work was done when the second author was on academic leave, visiting Florida Institute of Technology. The financial support of The Scientific and Technological Research Council of Turkey (TUBITAK) is gratefully acknowledged.

\section{References}

[1] D. D. Bainov and P. S. Simeonov, Oscillation Theory of Impulsive Differential Equations, International Publications, Orlando, Fla, USA, 1998.

[2] D. D. Bă̌nov and P. S. Simeonov, Impulsive Differential Equations: Asymptotic Properties of the Solutions, vol. 28 of Series on Advances in Mathematics for Applied Sciences, World Scientific, Singapore, 1995.

[3] D. Bainov and V. Covachev, Impulsive Differential Equations with a Small Parameter, vol. 24 of Series on Advances in Mathematics for Applied Sciences, World Scientific, Singapore, 1994.

[4] D. D. Bainov and P. S. Simeonov, Impulsive Differential Equations: Periodic Solutions and Applications, Longman Scientific and Technical, Harlow, UK, 1993.

[5] V. Lakshmikantham, D. D. Bainov, and P. S. Simeonov, Theory of Impulsive Differential Equations, vol. 6 of Series in Modern Applied Mathematics, World Scientific, Singapore, 1989.

[6] A. M. Samoilenko and N. A. Perestyuk, Impulsive Differential Equations, vol. 14 of World Scientific Series on Nonlinear Science. Series A: Monographs and Treatises, World Scientific, Singapore, 1995.

[7] E. Kruger-Thiemer, "Formal theory of drug dosage regiments I," Journal of Theoretical Biology, vol. 13, pp. 212-235, 1966.

[8] J. G. Pierce and A. Schumitzky, "Optimal impulsive control of compartment models. I. Qualitative aspects," Journal of Optimization Theory and Applications, vol. 18, no. 4, pp. 537-554, 1976.

[9] K. Gopalsamy and B. G. Zhang, "On delay differential equations with impulses," Journal of Mathematical Analysis and Applications, vol. 139, no. 1, pp. 110-122, 1989. 
[10] D. D. Bainov, Y. I. Domshlak, and P. S. Simeonov, "Sturmian comparison theory for impulsive differential inequalities and equations," Archiv der Mathematik, vol. 67, no. 1, pp. 35-49, 1996.

[11] S. Wu and Y. Duan, "Oscillation, stability, and boundedness of second-order differential systems with random impulses," Computers \& Mathematics with Applications, vol. 49, no. 9-10, pp. 1375-1386, 2005.

[12] R. P. Agarwal, S. R. Grace, and D. O’Regan, Oscillation Theory for Second Order Dynamic Equations, vol. 5 of Series in Mathematical Analysis and Applications, Taylor' Francis, London, UK, 2003.

[13] E. Hille, "Non-oscillation theorems," Transactions of the American Mathematical Society, vol. 64, pp. 234$252,1948$.

[14] W. Leighton, "On self-adjoint differential equations of second order," Journal of the London Mathematical Society, vol. 27, pp. 37-47, 1952.

[15] F. V. Atkinson, “On second-order non-linear oscillations," Pacific Journal of Mathematics, vol. 5, pp. 643-647, 1955.

[16] A. Özbekler and A. Zafer, "Sturmian comparison theory for linear and half-linear impulsive differential equations," Nonlinear Analysis: Theory, Methods E Applications, vol. 63, no. 5-7, pp. 289297, 2005.

[17] A. Özbekler and A. Zafer, "Picone's formula for linear non-selfadjoint impulsive differential equations," Journal of Mathematical Analysis and Applications, vol. 319, no. 2, pp. 410-423, 2006.

[18] A. Özbekler and A. Zafer, "Forced oscillation of super-half-linear impulsive differential equations," Computers \& Mathematics with Applications, vol. 54, no. 6, pp. 785-792, 2007.

[19] J. Shen, "Qualitative properties of solutions of second-order linear ODE with impulses," Mathematical and Computer Modelling, vol. 40, no. 3-4, pp. 337-344, 2004.

[20] Z. Luo and J. Shen, "Oscillation of second order linear differential equations with impulses," Applied Mathematics Letters, vol. 20, no. 1, pp. 75-81, 2007.

[21] C. Huang, "Oscillation and nonoscillation for second order linear impulsive differential equations," Journal of Mathematical Analysis and Applications, vol. 214, no. 2, pp. 378-394, 1997.

[22] A. Wan and W. Mao, "Oscillation and asymptotic stability behavior of a third order linear impulsive equation," Journal of Applied Mathematics E Computing, vol. 18, no. 1-2, pp. 405-417, 2005.

[23] Y. S. Chen and W. Z. Feng, "Oscillations of higher-order linear ODEs with impulses," Journal of South China Normal University. Natural Science Edition, no. 3, pp. 14-19, 2003.

[24] W. Feng, "Oscillations of fourth order ODE with impulses," Annals of Differential Equations, vol. 19, no. 2, pp. 136-145, 2003.

[25] Y. S. Chen and W. Z. Feng, "Oscillations of second order nonlinear ODE with impulses," Journal of Mathematical Analysis and Applications, vol. 210, no. 1, pp. 150-169, 1997.

[26] G. H. Hardy, J. E. Littlewood, and G. Pólya, Inequalities, Cambridge University Press, Cambridge, UK, 1934.

[27] X. Liu and Z. Xu, "Oscillation of a forced super-linear second order differential equation with impulses," Computers $\mathcal{E}$ Mathematics with Applications, vol. 53, no. 11, pp. 1740-1749, 2007.

[28] J. R. Graef and J. Karsai, "Intermittant and impulsive effects in second order systems," Nonlinear Analysis: Theory, Methods \& Applications, vol. 30, pp. 1561-1571, 1997.

[29] J. R. Graef and J. Karsai, "Oscillation and nonoscillation in nonlinear impulsive systems with increasing energy," in Proceedings of the International Conference on Dynamical Systems and Differential Equations, Atlanta, Ga, USA, 2000.

[30] J. R. Graef and J. Karsai, "On the oscillation of impulsively damped halflinear oscillators," in Proceedings of the 6th Colloquium on the Qualitative Theory of Differential Equations (Szeged, '99), pp. 1-12, Electronic Journal of Qualitative Theory of Differential Equations, 2000.

[31] A. Özbekler and A. Zafer, "Interval criteria for the forced oscillation of super-half-linear differential equations under impulse effects," Mathematical and Computer Modelling, vol. 50, no. 1-2, pp. 59-65, 2009.

[32] J. Luo and L. Debnath, "Oscillations of second-order nonlinear ordinary differential equations with impulses," Journal of Mathematical Analysis and Applications, vol. 240, no. 1, pp. 105-114, 1999.

[33] W. Xiu-li, C. Si-Yang, and H. Ji, "Oscillation of a class of second-order nonlinear ODE with impulses," Applied Mathematics and Computation, vol. 138, no. 2-3, pp. 181-188, 2003.

[34] J. Luo, "Second-order quasilinear oscillation with impulses," Computers \& Mathematics with Applications, vol. 46, no. 2-3, pp. 279-291, 2003. 
[35] Z. He and W. Ge, "Oscillations of second-order nonlinear impulsive ordinary differential equations," Journal of Computational and Applied Mathematics, vol. 158, no. 2, pp. 397-406, 2003.

[36] A. Özbekler, "Oscillation criteria for second order nonlinear impulsive differential equations, further progress in analysis," in Proceedings of the 6th International ISAAC Congress, pp. 545-554, World Scientific, Ankara, Turkey, 2009.

[37] C. Zhang and W. Feng, "Oscillation for higher order nonlinear ordinary differential equations with impulses," Electronic Journal of Differential Equations, no. 18, pp. 1-12, 2006.

[38] L. J. Pan, G. Q. Wang, and S. S. Cheng, "Oscillation of even order nonlinear differential equations with impulses," Funkcialaj Ekvacioj, vol. 50, no. 1, pp. 117-131, 2007.

[39] M. B. Dimitrova, "Nonoscillation of the solutions of impulsive differential equations of third order," Journal of Applied Mathematics and Stochastic Analysis, vol. 14, no. 3, pp. 309-316, 2001.

[40] X. Fu and X. Li, "Oscillation of higher order impulsive differential equations of mixed type with constant argument at fixed time," Mathematical and Computer Modelling, vol. 48, no. 5-6, pp. 776-786, 2008. 